EPJ Web of Conferences 22, 00009 (2012)

DOI: $10.1051 /$ epjconf/20122200009

(C) Owned by the authors, published by EDP Sciences, 2012

\title{
An introduction to the tools hosted in the Bilbao Crystallographic Server
}

\author{
E.S. Tasci, G. de la Flor, D. Orobengoa, C. Capillas, J.M. Perez-Mato \\ and M.I. Aroyo \\ Departamento Física de la Materia Condensada, Facultad de Ciencia y Tecnología, \\ Universidad del País Vasco, Apto. 644, 48080 Bilbao, Spain
}

\begin{abstract}
The programs hosted in the Bilbao Crystallographic Server (http://www.cryst.ehu.es) are briefly explained along with worked examples on various cases related to different fields of applications. It is our aim to have this text acting as a primer on the various usage of the crystallographic tools in conjunction with each other due to the modular structure of the server. For this reason, diverse topics such as crystallographic groups and their subgroups, pseudosymmetry, extinction conditions, $\mathbf{k}$-vectors and irreducible representations have been discussed in the context.
\end{abstract}

\section{GENERAL LAYOUT OF THE SERVER}

The Bilbao Crystallographic Server (http://www.cryst.ehu.es) [1, 2] hosts databases and programs (also called "modules" or "tools") related to group theory, crystallography and solid-state physics applications. The server is built on a database core, which includes data from International Tables for Crystallography, Volume A: Space-group symmetry (hereafter referred to as ITA) [3], and the data on maximal subgroups of space groups as listed in Part 1 of International Tables for Crystallography, Volume A1: Symmetry relations between space groups (hereafter referred to as ITA1) [4]. There is an access to the crystallographic data for the subperiodic layer and rod groups (International Tables for Crystallography, Volume E: Subperiodic groups (hereafter referred to as ITE) [5]) and their maximal subgroups. A database on incommensurate structures incorporating modulated structures and composites, and a k-vector database with Brillouin-zone figures and classification tables of the wave vectors for space groups are also available. The different programs of the server form the so-called "shells" where they are gathered with respect to a common application field. Since its launch in 1997, the server has extended its capabilities as is evident from the number of diverse shells and programs.

The programs in the server are designed to incorporate the results of the other programs consecutively. The main idea behind this modular approach is to customize the offered services by combining and merging related modules in accordance with their needs.

In the following sections, we will give a general description of each shell, along with brief explanations of the tools contained within and examples demonstrating the usage of a collection of these tools. The last section contains basic facts and definitions on some selected topics of mathematical crystallography necessary for the description of the databases and programs of the Bilbao Crystallographic Server treated in this article.

\section{SHELLS}

\subsection{Space groups retrieval tools}

This shell hosts the tools related to the space groups, in general. It can be thought as an interface to tabulated data and thus, usually only the sequential space group number is necessary to retrieve the desired information. Most of the data have been compiled from ITA but in addition to the printed

This is an Open Access article distributed under the terms of the Creative Commons Attribution-Noncommercial License 3.0, which permits unrestricted use, distribution, and reproduction in any noncommercial medium, provided the original work is properly cited. 
version, acquisition of data with respect to user-defined settings is also supported. The programs and databases of the Bilbao Crystallographic Server use the so-called standard or default settings. These are specific settings of space groups that coincide with the conventional space-group descriptions found in ITA. For space groups with more than one description in ITA, the following settings are chosen as standard: unique axis $b$ setting, cell choice 1 for monoclinic groups, hexagonal axes setting for rhombohedral groups, and origin choice 2 (origin in $\overline{1}$ ) for the centrosymmetric groups listed with respect to two origins in ITA.

Generators and/or general positions of a space group in different settings are listed via GENPOS. For this purpose the only necessary input is the sequential number of the space group (where, if unknown, can be identified by choosing the corresponding Hermann-Mauguin symbol). In the output, the generators/general positions are listed by their coordinate triplets and $(3 \times 4)$ matrix-column pairs accompanied by their geometric interpretation as well ( $c f$. Section 4.1). Apart from the standard-setting option one can also have the listing in another desired setting by specifying its transformation with respect to the standard one ( $c f$. Section 4.2). The optional ITA settings include the 530 non-conventional settings of monoclinic and orthorhombic space groups listed in Table 4.3.2.1 of ITA. The geometric interpretations of the symmetry operations (belonging to either standard or non-conventional settings) can also be calculated independently via the SYMMETRY OPERATIONS module.

WYCKPOS lists the Wyckoff positions for a designated space group along with their Wyckoff letters, multiplicities and oriented symbols of the site-symmetry groups. One can also have the listing in another desired setting by defining the corresponding transformation matrix or choosing it from the IT A designated settings.

HKLCOND gives access to the reflection conditions which, following ITA, are formulated as conditions of occurrence. Accepting the space group number as input, the program returns the tabulated data of the so-called general reflection conditions (that apply to all Wyckoff positions), as well as the special or extra conditions valid only for specific Wyckoff positions (in addition to the general ones).

NORMALIZER module lists the Euclidean and affine normalizers of a given space group, described by sets of additional symmetry operations that generate the normalizers successively from the space groups. The parametric presentation of the matrix-column pairs of the elements of the affine normalizers of triclinic and monoclinic groups (which are not isomorphic to groups of motions) follow closely the listing of ITA, Chapter 15. Optionally, the program also calculates the Euclidean normalizers of enhanced symmetry for the cases of specialized metric of triclinic, monoclinic and some orthorhombic space groups.

The assignment of Wyckoff positions to Wyckoff sets is shown by WYCKSETS: Wyckoff positions of a given space group, equivalent with respect to the affine normalizer, are listed together along with their Wyckoff letter, multiplicities, site-symmetry groups and a coordinate triplet of an orbit representative. In a sequential page, the possible transformations among equivalent Wyckoff positions are displayed with respect to normalizer transformations.

MAXSUB gives access to the database of maximal subgroups of space groups which includes data from ITA1. For a designated space group $\mathcal{G}$, MAXSUB lists the related maximal subgroups $\mathcal{H}$ of indices up to index 9 . Each subgroup $\mathcal{H}$ is specified by its ITA-number, the index in the group $\mathcal{G}$ and the transformation matrix-column pair $(\boldsymbol{P}, \boldsymbol{p})$ that relates the standard bases of $\mathcal{H}$ and $\mathcal{G}$ ( $c f$. Section 4.3.2). The different maximal subgroups are distributed in classes of conjugate subgroups. For certain applications it is necessary to represent the subgroups $\mathcal{H}$ as subsets of the elements of $\mathcal{G}$. This is achieved by an option in MAXSUB which transforms the general-position representatives of $\mathcal{H}$ by the corresponding matrix-column pair $(\boldsymbol{P}, \boldsymbol{p})^{-1}$ to the coordinate system of $\mathcal{G}$. A link provides the splittings of all Wyckoff positions of $\mathcal{G}$ with respect to those of $\mathcal{H}$.

The maximal isomorphic subgroups are infinite in number and have indices of $p, p^{2}$ or $p^{3}$, where $p$ is a prime. SERIES lists the series of maximal isomorphic subgroups upto and including, by default, index 27 (125 for cubic groups) along with the parametric description of the series as given in ITA1. The default upper limit for the indices can be overridden using the option for defining the maximum index, as well as filtering the listing for a specified index only. 


\section{Contribution of Symmetries in Condensed Matter}

The tool MINSUP lists the isomorphic and non-isomorphic minimal supergroups $\mathcal{S}$ of indices 2, 3 and 4 for any space group $\mathcal{G}$. Each supergroup is specified by the transformation matrix relating the standard bases of $\mathcal{S}$ and $\mathcal{G}$ with additional links to the tools WYCKSPLIT and COSETS. For the triclinic and monoclinic space groups the lists include minimal isomorphic supergroups of indices up to 7 with an optional link for online calculations of minimal isomorphic supergroups of higher indices.

\subsection{Brillouin-zone database}

A decisive role in the description and classification of space-group representations is played by the wave or k-vectors which are described as vectors in reciprocal space. They can be classified according to their symmetry properties by the so-called reciprocal-space groups [6], which are isomorphic to symmorphic space groups. This isomorphism allows the application of crystallographic conventions in the classification of the wave vectors (and henceforth in the space-group representations). For example, the different symmetry types of k-vectors correspond to the different kinds of point orbits (Wyckoff positions) in the symmorphic space groups; the unit cells with the asymmetric units given in ITA can serve as Brillouin zones and representation domains, etc. (For a review of the reciprocal space-group approach, the reader is referred to Refs. [7, 8] and the references therein).

The wave-vector database of the Bilbao Crystallographic Server contains Brillouin-zone figures and wave-vector tables for all 230 space groups. In this compilation, the figures and wave-vector data based on the reciprocal-space group approach are compared with the representation domains and the lists of special k-vector in the tables on space-group representations by Cracknell, Davies, Miller and Love [9] (hereafter referred to as CDML). One can access to the database through the program KVEC. The k-vector database uses the ITA-number of the space group as input. In the output figures, the Brillouin zones of CDML and the conventional unit cells of ITA are displayed. The asymmetric units are chosen often in analogy to those of ITA. In the accompanying tables, the k-vector data as listed by CDML are compared with the Wyckoff-position description as given in ITA. There are several sets of figures and tables for the same space group when its Brillouin-zone shape depends on the lattice parameters of the reciprocal lattice. The $\mathbf{k}$-vector data are the same for space groups of the same arithmetic crystal class.

\subsection{Subperiodic groups: layer, rod and frieze groups retrieval tools}

Aside from the subgroups of space groups with three dimensional lattices, there also exist subgroups called subperiodic with lattices of dimensions one or two. Layer groups are two-periodic groups acting on three dimensional space, while rod groups are one-periodic groups acting on three dimensional space. This shell holds the tools accessing the database for layer and rod groups which includes data from ITE. The data for the frieze groups (one-periodic groups acting on two dimensional space) are currently being integrated to the subperiodic database.

GENPOS lists the $(3 \times 4)$ matrix-column pairs of the generators and the symmetry operations of the general positions of the layer and rod groups including also their geometrical interpretations. The user can work in the conventional setting as well as in a non-conventional setting by providing the transformation matrix relating the non-conventional setting to the conventional one.

WPOS retrieves the Wyckoff positions of a given layer or rod group along with its Wyckoff letter, multiplicity, orientated symbols of the site symmetry groups and the coordinate triplets of the orbit unit-cell representatives.

For a given layer or rod group, MAXSUB lists all isotypic and non-isotypic maximal subgroups of indices 2, 3 and 4 along with their indices and transformation matrices.

\subsection{Group-subgroup relations of space groups}

The necessary tools that perform calculations related to a pair of group $\mathcal{G}$ and subgroup $\mathcal{H}$ are contained in this shell. Because of this nature, the input usually consists of the group and subgroup space group 
numbers and the index. For certain applications, instead of the index, it is necessary to input the transformation matrix that relates the group to the subgroup standard bases.

For a given group-subgroup pair $\mathcal{G}>\mathcal{H}$, SUBGROUPGRAPH explores the relationship between the space-group types $\mathcal{G}$ and $\mathcal{H}$ by constructing all possible group-subgroup chains of intermediate maximal subgroups $\mathcal{Z}_{k}: \mathcal{G}>\mathcal{Z}_{1}>\ldots>\mathcal{Z}_{n}=\mathcal{H}$ as presented in Fig. 1 . For a specified index of $\mathcal{H}$ in $\mathcal{G}$, there are, in general, a number of different subgroups $\mathcal{H}_{j}<\mathcal{G}$ isomorphic to $\mathcal{H}$. The program retrieve all possible chains of maximal subgroups that relate all possible group-subgroup pairs $\mathcal{G}>\mathcal{H}_{j}$ with the corresponding transformation matrices $\left(\boldsymbol{P}_{j}, \boldsymbol{p}_{j}\right)$ ( $c f$. Section 4.3.2). One can also have the graph $\mathcal{G}>\mathcal{H}_{j}$ drawn as a maximal-subgroup diagram. For further details on SUBGROUPGRAPH, refer to Ref. [10].

Consider a group-subgroup pair $\mathcal{G}>\mathcal{H}$, with $\mathcal{H}$ a general subgroup of index [i]. The existence and the uniqueness of Hermann's group $\mathcal{M}$ [11] for any specific group-subgroup pair $\mathcal{G}>\mathcal{H}_{j}$ of index $[i], \mathcal{G}>\mathcal{M}>\mathcal{H}_{j}$ implies the possibility of factorizing any group-subgroup chain $\mathcal{G}>\mathcal{H}_{j}$ (and as a result, the total graph $\mathcal{G}>\mathcal{H}_{j}$ ) into two subchains (or subgraphs) of smaller indices: the translationengleiche or $t$-chain $\mathcal{G}>\mathcal{M}$ of index $\left[i_{P}\right]$, and the klassengleiche, or $k$-chain $\mathcal{M}>\mathcal{H}_{j}$ of index $\left[i_{L}\right]$ (cf. Section 4.3.2). The HERMANN tool identifies the Hermann groups for all subgroups $\mathcal{H}_{j}$ of $\mathcal{G}$ of a given index [i]. Similar to the SUBGROUPGRAPH output, the different subgroups $\mathcal{H}_{j}$ are distributed into conjugacy classes with additional links to tools for the splittings of the Wyckoff positions for $\mathcal{G}>\mathcal{H}$, or to the symmetry modes analysis of the possible phase-transition mechanism related to the symmetry break $\mathcal{G} \longrightarrow \mathcal{H}$.

In general, there exists a variety of possible specific group-subgroup relationships between two space-group types $\mathcal{G}$ and $\mathcal{H}$ that form a group-subgroup pair $\mathcal{G}>\mathcal{H}$. One essential characteristic of such group-subgroup relationship is the index $[i]=\left[i_{P}\right] \cdot\left[i_{L}\right]$ of $\mathcal{H}$ in $\mathcal{G}$. Given the space-group types of $\mathcal{G}$ and $\mathcal{H}$ and the multiplication the unit cell $\left[i_{L}\right]$ (expressed also by the ratio of the structure formula units of $\mathcal{G}$ and $\mathcal{H}$ ), the tool INDEX returns the possible index [i] of $\mathcal{H}$ in $\mathcal{G}$, compatible with the given $\left[i_{L}\right]$. After retrieving the index, one can directly proceed to the corresponding subgroup data (maximalsubgroup graph and transformation matrices) using the optional link.

For a given group-subgroup pair $\mathcal{G}>\mathcal{H}$ and the transformation matrix $(\boldsymbol{P}, \boldsymbol{p})$ relating them, the COSETS tool performs the calculation of left/right coset decomposition of $\mathcal{G}$ with respect to $\mathcal{H}$.

A crystallographic orbit of symmetrically-equivalent atoms with respect to $\mathcal{G}$ may split into suborbits of symmetrically-equivalent atoms with respect to a subgroup $\mathcal{H}<\mathcal{G}$. By supplying the group and subgroup space-group numbers and the relating transformation matrix $(\boldsymbol{P}, \boldsymbol{p})$, WYCKSPLIT lists the splittings of the Wyckoff positions of $\mathcal{G}$ into Wyckoff positions of the subgroup $\mathcal{H}<\mathcal{G}$ along with a detailed correspondence table between the representatives of the orbits of the group and the subgroup. For further details, the reader can refer to Ref. [12].

Given the space-group types of $\mathcal{S}$ and $\mathcal{G}$ and their index, the program SUPERGROUPS calculates all different supergroups $\mathcal{S}_{j}>\mathcal{G}$ isomorphic to $\mathcal{S}$ and of the same index. The calculation is done by inverting the subgroup data for $\mathcal{S}_{j}>\mathcal{G}$ obtained by SUBGROUPGRAPH and applying the so-called normalizer procedure. Optionally one can choose between Euclidean or affine normalizers for $\mathcal{S}$ and $\mathcal{G}$. Similar to the MINSUP output, each supergroup is specified by the transformation matrix $(\boldsymbol{P}, \boldsymbol{p})$ relating the standard bases of $\mathcal{S}$ and $\mathcal{G}$ with optional links to the tools WYCKSPLIT and COSETS. Additional details can be found in Ref. [13].

The program CELLSUB determines all subgroups of a given space group $\mathcal{G}$ that are compatible with a specific multiple of the unit cell of $\mathcal{G}$, i.e. with a given $\left[i_{L}\right]$ index. Furthermore, the program also classifies these subgroups in accordance with their type (i.e. translationengleiche, klassengleiche or general). From the list, one can proceed further to the classification in conjugate classes of subgroups. Using the main interface for CELLSUB, the results for a given $\left[i_{L}\right]$ index can be filtered with respect to the possible point group, crystal system, centering and/or types (polar, non-polar, axial, non-axial, ferroelastic) of the subgroups. Further details on the program and examples of its application can be found in the article on symmetry considerations of structural phase transitions of this volume [14]. 


\section{Contribution of Symmetries in Condensed Matter}

Operating in a similar context as the CELLSUB tool, CELLSUPER instead retrieves the list of supergroups whose $\left[i_{L}\right]$-indices are upto and including the designated index limit. It has the same filtering options as of CELLSUB.

The program COMMONSUBS [15] studies the possible relationships via common subgroup $\mathcal{H}$ between two space groups $\mathcal{G}_{1}$ and $\mathcal{G}_{2}$ that do not form a group-subgroup pair, i.e. $\mathcal{G}_{1}>\mathcal{H}<\mathcal{G}_{2}$. In general, there exists an infinite number of common subgroups of $\mathcal{G}_{1}$ and $\mathcal{G}_{2}$ and in COMMONSUBS the subgroup search is restricted by two conditions: (i) introducing a maximal value $\left[\left(i_{L}\right)_{\max }\right]$ that limits the cell multiplication of $\mathcal{H}$ with respect to $\mathcal{G}_{1}$ and $\mathcal{G}_{2}$ (and thus limits the indices $\left[i_{1}\right]$ and $\left[i_{2}\right]$ of $\mathcal{H}$ in $\mathcal{G}_{1}$ and $\mathcal{G}_{2}$ ); (ii) as the main application of COMMONSUBS is related to the comparison of two structures with space groups $\mathcal{G}_{1}$ and $\mathcal{G}_{2}$ within a common subgroup $\mathcal{H}$, it is reasonable to require that the number of formula units $Z_{H}$ in the unit cell of $\mathcal{H}$ is the same for both structures. Given the space group types $\mathcal{G}_{1}$ and $\mathcal{G}_{2}$ with the corresponding numbers of formula units and the limiting index $\left[\left(i_{L}\right)_{\text {max }}\right]$, the program returns the list of common subgroups $\mathcal{H}$ specified by their Hermann-Mauguin symbols, the point groups $\mathcal{P}_{\mathcal{H}}$, the indices $\left[i_{1}\right]$ and $\left[i_{2}\right]$ of $\mathcal{H}$ in the space groups $\mathcal{G}_{1}$ and $\mathcal{G}_{2}$, and the corresponding factors $\left[i_{P}\right]$ and $\left[i_{L}\right]$. The search of common subgroups can be further restricted by specifying the point group, the crystal class, or the type of centring for the common subgroup. The generalization of the procedure for the case of common subgroups of three groups $\mathcal{G}$ is straightforward and has also been implemented in the program COMMONSUBS.

Given the sequential numbers of two space groups and the number of formula units, along with a limit on the maximal index $\left[\left(i_{L}\right)_{\max }\right]$, COMMONSUPER returns the list of the common supergroups for these space groups. The result also contains the indices [i] (and their factorization into the translationengleiche and klassengleiche components $[i]=\left[i_{P}\right] \cdot\left[i_{L}\right]$ ) along with optional links to the transformation matrices relating the common supergroups to the given space groups.

Each crystallographic orbit of symmetrically-equivalent points of a space group $\mathcal{G}$ is characterized by its inherent or eigensymmetry group $\mathcal{E}$. The orbit is called characteristic if $\mathcal{E}=\mathcal{G}$ or non-characteristic if the eigensymmetry group is a supergroup of $\mathcal{G}, \mathcal{E}>\mathcal{G}$. For a given Wyckoff position of a space group $\mathcal{G}$, the program NONCHAR calculates the eigensymmetry groups of the crystallographic orbits of $\mathcal{G}$ assigned to the specified Wyckoff position. Further, the tool determines possible non-characteristic orbits and their eigensymmetry groups for specialized values of the variable parameters of the Wyckoff position coordinate triplets and/or for speciliazed metric of $\mathcal{G}$. The eigensymmetry groups $\mathcal{E}>\mathcal{G}$ are specified by their Hermann-Mauguin symbols, indices of $\mathcal{G}$ in $\mathcal{E}$ and the corresponding transformation matrices $(\boldsymbol{P}, \boldsymbol{p})$.

\subsection{Representation theory applications}

The representations of crystallographic groups and related data play an important role in analysis of crystallographic, solid-state physics and structure-chemistry problems. The applications that are dedicated to representations of crystallographic space and point groups are collected within this shell. They employ the irreducible representations (or irreps for short) of crystallographic space and point groups and the relations between them.

For further information on irreps of crystallographic groups and the procedures of calculations, the reader is referred to Refs. $[1,9,16]$.

REPRES calculates the irreps of space groups following a general scheme based on a normalsubgroup induction method of constructing the irreps of a group $\mathcal{G}$ starting from those of its translation subgroup $T_{\mathcal{G}}$ which is a normal subgroup of $\mathcal{G}, \mathcal{H} \triangleleft \mathcal{G}$. As input, the sequential space group number and the wave or k-vector (which can be selected from the table related to the space group or can be introduced independently, supporting both primitive and conventional dual bases) are given. As output it returns the information on the space group $\mathcal{G}$ (lists of the non-translational generators and translational coset representatives of $\mathcal{G}$ ), the $\mathbf{k}$-vector and the arms of the star of the $\mathbf{k}$-vector in primitive and conventional dual bases and the matrices of the little-group irreps for the translational 


\section{EPJ Web of Conferences}

coset representatives of little group $\mathcal{G}^{\mathbf{k}}$ of the wave vector. The matrices of the full-group irreps for the non-translation generators of the space group are presented in block-matrix form: the induction matrices and the corresponding blocks of little group representation matrices are listed separately by the program.

DIRPRO tool lists the direct (Kronecker) products of the irreps of a space group for a given set of two k-vectors, along with their associated little groups.

For a given k-vector, CORREL calculates the correlations between the irreps of a group-subgroup pair $\mathcal{G}>\mathcal{H}$, related by the input transformation matrix $(\boldsymbol{P}, \boldsymbol{p})$.

POINT lists the irreps of the specified point group among the 32 crystallographic point groups. Along with the international (Hermann-Mauguin) and Schoenflies symbols for the given point group, the character table, the subgroups of the specified point group, the multiplication table of its irreps, their tensor representations, selection rules for fundamental transitions and the subduction from the rotation group irreps are retrieved.

The program SITESYM calculates the multiplicities of space-group irreps in the representations induced by the irreps of a site-symmetry group. For a given space group, wave vector and a selected representative of a Wyckoff position, SITESYM returns the symmetry operations of the site symmetry group and its irreps. Using the Frobenius reciprocity theorem, the program calculates the multiplicities of the space-group irreps for the specified wave vector from the decompositions of the subduced representations of the site-symmetry groups.

\subsection{Structure utilities}

The utilities contained in this shell are mostly concerned with a single structure and offer visualization, assignment of Wyckoff positions as well as transformation under general or specific conditions.

The server's default structure definition format is the so-called $B C S$ file format where sequential space group number, the lattice parameters, number of atoms in the asymmetric unit is followed by atomic species, Wyckoff position and coordinates of the representative atoms for each orbit in fractional (direct) format. Comments can be included by inserting "\#” sign at the beginning of lines. A sample structure is presented in this format in Table 8.

Most of the structure utility programs assume that the given structure descriptions are referred to the standard settings of the space groups. The possibility to use non-standard settings by some programs is explicitly stated on the initial page of the programs. In addition to the $B C S$ file format, most of the tools also support the standard CIF format [17] for input/output where applicable.

CELLTRAN is used to transform the lattice parameters of a given unit cell, in accordance with the specified transformation matrix ( $c f$. Section 4.2). As a result, the metric tensors (in both conventional and primitive bases) for the initial and transformed unit cell are calculated. In addition, the program provides data of the reduced unit cell and the standard root tensor [18] relating the conventional and Cartesian bases.

STRAIN compares two given unit cells (defined in terms of their lattice parameters) in detail by constructing their metric tensors and calculating the (linear and finite) Lagrangian strain tensors [19]. A quantitative measure of the similarity of the two lattices is given by the so-called degree of lattice distortion [20] which is calculated from the eigenvalues of the strain tensor.

Considering the fractional atomic coordinates and the space group number of a given structure, WPASSIGN calculates the Wyckoff positions corresponding to the designated atomic positions for each atom. It also lists the site-symmetry group along with the coordinate triplets of the unit-cell representatives of the atomic orbit as well. The program accepts CIF files as input along with the standard $B C S$ file format.

TRANSTRU transforms a given structure using a given transformation matrix $(\boldsymbol{P}, \boldsymbol{p})$ and optionally a low symmetry space group number. If only $(\boldsymbol{P}, \boldsymbol{p})$ is given, the structure's lattice parameters and atomic positions will be transformed but no compatibility check or Wyckoff position assignment will take place. However, if the transformation is performed with the knowledge of the subgroup, the validity of the transformation matrix is checked and the subgroup Wyckoff positions assignments are also performed. 


\section{Contribution of Symmetries in Condensed Matter}

The program SETSTRU performs coordinate transformations of a given structure between any of the alternative ITA settings of its space group. For a space group with several ITA settings, SETSTRU asks the user to select a setting for the initial structure followed by the initial structure data (i.e. cell parameters and atomic positions). Via the "transform to" option, the user can transform this structure directly to the standard or any other alternative non-standard setting.

Given a structure, EQUIVSTRU returns all of its equivalent descriptions [21], i.e. all possible descriptions with respect to the same space-group setting and origin. The equivalent descriptions are obtained by transforming the initial one with respect to the Euclidean normalizers of the structure's space group. In the case of specialized metric, the program uses the enhanced Euclidean normalizer which is calculated via the NORMALIZER module.

WPASSIGN, TRANSTRU, SETSTRU and EQUIVSTRU modules can also generate the output structure data in CIF format, aside from the $B C S$ file format. One can then submit these structures to the VISUALIZE tool where they are visualized in a 3D-interactive panel using the visualization package Jmol [22].

The program COMPSTRU compares two descriptions of the same structure obtained with respect to the same standard setting of the symmetry space group. Keeping one of the descriptions as a reference, the program searches through all possible equivalent descriptions of the second one (EQUIVSTRU) to find the Euclidean normalizer transformation (or enhanced Euclidean in case of specialized metric) such that the coordinates of the corresponding atoms in both descriptions match within a given tolerance. The program is especially useful when one has to decide if two different structural models of the same compound (e.g. coming from different sources) indeed represent different phases or not. The maximum displacements $\Delta$ between the corresponding atoms of the two structural models serve for the quantitative evaluation of their similarity.

\subsection{Solid state theory applications}

This shell contains the tools facilitating the study of specific problems related to solid-state physics and structural chemistry with special relevance to the analysis of phase transitions.

Given a structure specified by its symmetry space group (ITA-number) and by the Wyckoff positions of the occupied orbits of atoms, SAM computes the symmetry-adapted modes in the $\Gamma(0,0,0)$ point and analyzes them for infrared and Raman activity. The symmetry modes, classified according to the irreps of the point group of the crystal are calculated starting from the corresponding permutational and mechanical representations, and applying the projection-method technique. In addition, useful data on the point group irreps such as character tables, multiplication tables of irreps, irrep-decompositions of some important representations of the point group, etc. are presented. Details on the procedure is available from Ref. [23].

NEUTRON calculates the phonon extinction rules for inelastic neutron scattering as well as for thermal diffuse scattering with respect to the input $\mathbf{k}$-vector and sequential space group number. In the output, special $\{\mathrm{h}, \mathrm{k}, 1\}$ conditions are listed for which extinctions are observed depending on the irrep associated with the phonon mode. For more information, the reader is referred to Ref. [24].

SYMMODES is used for the group-theoretical study of continuous or quasicontinuous displacive phase transitions. These phase transitions are characterized by a group-subgroup relation of the space groups $G>H$ describing the symmetry of the two phases. This symmetry break can be explained by the freezing of one or several modes of the high symmetry structure, the primary modes. Other, secondary modes can also be present on the symmetry break due to their coupling to the primary ones. SYMMODES calculates all these modes together with a detailed group-subgroup description of the $\mathcal{G}>\mathcal{H}$ chain. For more information on SYMMODES, refer to Ref. [25].

Given the structures of two phases of high and low symmetry related by a displacive phase transition and based on the information calculated with the program SYMMODES, AMPLIMODES carries out the symmetry mode decomposition of the distortion relating the two phases. This analysis allows the calculation of the amplitudes and the polarization vectors of each of the distortion 
modes and their hierarchy in the transition mechanism. AMPLIMODES has been described in detail in Ref. [26].

Being one of the most complex tools in the Bilbao Crystallographic Server, PSEUDO utilizes many modules in the background in the search for structural pseudosymmetry. For a crystal structure, specified by its space group $\mathcal{G}$, the cell parameters and the coordinates of the atoms in the asymmetric unit, the program searches for a pseudosymmetry with respect to any supergroup $\mathcal{S}$ of the group $\mathcal{G}$. Optionally, PSEUDO can perform the search among the minimal supergroups (MINSUP) or supergroups related with a designated $\left[i_{L}\right]$-index (CELLSUPER) as well as for a specific supergroup denoted by its space group number and the transformation matrix $(\boldsymbol{P}, \boldsymbol{p})$. Additional metrical conditions restrict the list of possible supergroups for structures of triclinic and monoclinic symmetry. Depending on the type of search, single or multiple candidates of possible higher symmetry space groups $\mathcal{S}$ can be chosen from the provided supergroup list. The pseudosymmetry search is carried out by a straightforward checking the compatibility of the initial crystal structure under the additional symmetry operations of the supergroups $\mathcal{S}$. This is achived by the comparison of the initial structure with its variants obtained by transformations with the coset representatives of $\mathcal{S}$ relative to $\mathcal{G}$. A structure is considered pseudosymmetric if the distances between the initial and transformed positions of each atom are less than or equal to the designated maximum tolerance value. An extensive coverage of PSEUDO is available via the Refs. $[27,28]$.

DOPE calculates a quantitative value for pseudosymmetry based on the analysis of the symmetry properties of the electron density function. For a given space group, the minimal supergroups are processed to calculate the degrees of dissymmetry and pseudosymmetry, based on the structure factors obtained via the CCTBX library [29] and the representatives of the coset decomposition of the higher symmetry structure's space group with respect to the actual space group of the structure. For more details on DOPE and the electron density approach to pseudosymmetry search and its comparison with the atomic displacement method (applied in PSEUDO) can be found in Ref. [30].

For a given set of two structures that are not group-subgroup related, TRANPATH proceeds to find a hypothetical intermediate structure belonging to a common subgroup of the two given end phase structures, and then checks the compatibility in terms of lattice match (by calculating the strain tensor) and the necessary displacements of the atoms. The thresholds for these compatibilities are defined by the user along with the maximum $\left[i_{L}\right]$-index of the common subgroup. Detailed information on TRANPATH can be found in Ref. [15].

\subsection{B-IncStrDB: Bilbao incommensurate structures database}

Incommensurate structures are aperiodic structures where the atoms undergo a modulation (concerning the position, occupation, thermal motion, etc.) such that a Bravais lattice can not be defined. These structures are described defining a reference periodic structure, the modulation wave vector(s) and a set of atomic modulation functions. The symmetry of the structures is introduced by means of the so-called superspace formalism, using superspace symmetry groups.

The Bilbao Crystallographic Server hosts the B-IncStrDB (Bilbao Incommensurate Structures Database) that contains a considerable set of reported incommensurate structures. The users can search among these structures using various criteria and have the structures exported in CIF format. New published incommensurate structures can be directly uploaded by the user if the generalized CIF-like format employed by JANA2006 [31] is used.

\section{EXAMPLES}

\subsection{Identifying relations between space groups}

In this example, we will gather information concerning the space groups $P \overline{3} 1 c$ (No. 163) and $P 2 / c$ (No. 13). By submitting these numbers to GENPOS, we retrieve the lists of 12 and 4 translational coset representatives of the general positions for $P \overline{3} 1 c$ and $P 2 / c$. 


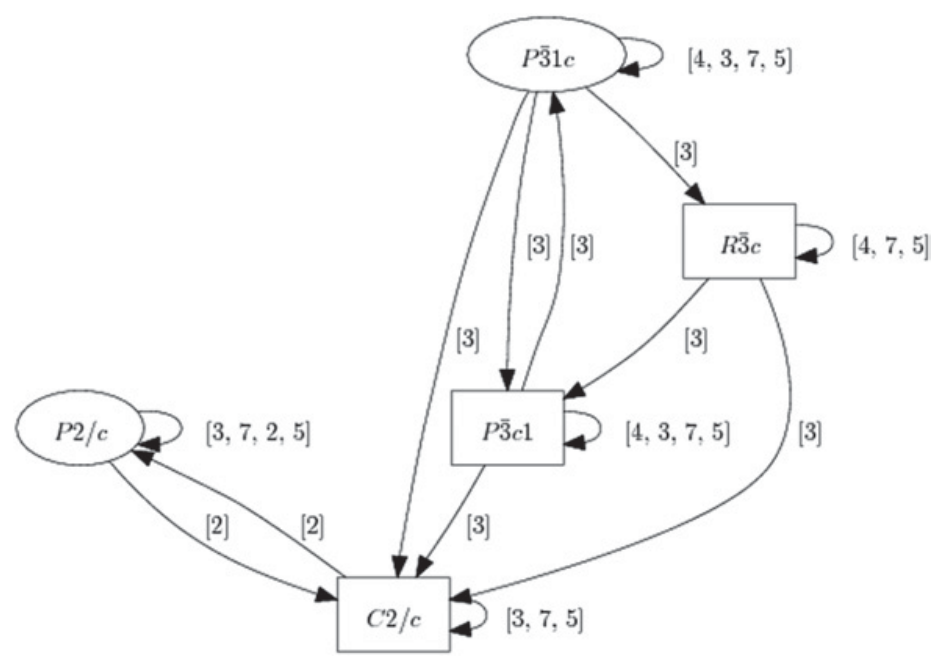

Figure 1. SUBGROUPGRAPH graph for possible group-subgroup relations between the space group types $P \overline{3} 1 c$ (No. 163) $\longleftrightarrow P 2 / c$ (No. 13).

Table 1. SUBGROUPGRAPH data for the description of the group-subgroup relations between the space group types $P \overline{3} 1 c$ (No. 163)

\begin{tabular}{|c|c|c|l|}
\hline No & Group & HM Symbol & Maximal Subgroups \\
\hline \hline 1 & 163 & $P \overline{3} 1 c$ & $015[3] ; 163[3] ; 163[4] ; 163[5] ; 163[7] ; 165[3] ; 167[3]$ \\
\hline 2 & 167 & $R \overline{3} c$ & $015[3] ; 165[3] ; 167[4] ; 167[5] ; 167[7]$ \\
\hline 3 & 165 & $P \overline{3} 1 c 1$ & $015[3] ; 163[3] ; 165[3] ; 165[4] ; 165[5] ; 165[7]$ \\
\hline 4 & 015 & $C 2 / c$ & $013[2] ; 015[3] ; 015[5] ; 015[7]$ \\
\hline 5 & 013 & $P 2 / c$ & $013[2] ; 013[3] ; 013[5] ; 013[7] ; 015[2]$ \\
\hline
\end{tabular}

Assuming that these two groups can be related, we proceed to SUBGROUPGRAPH, feeding their sequential space group numbers as input, leaving the index field blank. The returned table (Table 1) and graph (Fig. 1) describe the possible inter-relations between the two space-group types. For example, an edge connecting two vertices of the graph shows that the corresponding space groups form a groupmaximal subgroup pair of an index that is also indicated. Using the table and the graph, we can deduce that the shortest group-subgroup chain between $P \overline{3} 1 c$ and $P 2 / c$ is of index 6 and can be realized via the subgroup of the type $C 2 / c$.

Now that we have chosen the index, we can proceed to find the different specific subgroups $P 2 / c$ of $P \overline{3} 1 c$ and possible transformation matrices. A quick check made via the HERMANN module, reveals that $C 2 / c$ (No. 15) also acts as the Hermann group, being a translationengleiche subgroup of $P \overline{3} 1 c$ while being a klassengleiche supergroup of $P 2 / c$. Aside from this information, HERMANN also lists the possible transformation matrices for each of the present 2 classes of conjugate subgroups. In our case, each class holds 3 different subgroups and their related transformation matrices are also listed. These transformation matrices could also have been retrieved using SUBGROUPGRAPH and feeding the index as input.

We further proceed by selecting one of the transformation matrices, say " $c, a-b, a+b-c ; 0,-1 / 2,0$ " to be the one related to our case. With the two groups and the transformation matrix relating them, WYCKSPLIT returns the Wyckoff position splittings as rephrased in Table 2, while COSETS program calculates the coset decomposition of $P \overline{3} 1 c$ with respect to $P 2 / c$, as their representatives are presented in Table 3. 
Table 2. Wyckoff Positions Splitting for $P \overline{3} 1 c$ (No. 163) $\rightarrow P 2 / c$ (No. 13), transformation matrix: $\left(c, a-b, a+b-c ; 0,-\frac{1}{2}, 0\right)$ obtained by WYCKSPLIT.

\begin{tabular}{|c|c|c|}
\hline$\#$ & Group & Subgroup \\
\hline \hline 1 & $12 \mathrm{i}$ & $4 \mathrm{~g} 4 \mathrm{~g} 4 \mathrm{~g} 4 \mathrm{~g} 4 \mathrm{~g} 4 \mathrm{~g}$ \\
\hline 2 & $6 \mathrm{~h}$ & $2 \mathrm{f} 4 \mathrm{~g} 2 \mathrm{e} 4 \mathrm{~g}$ \\
\hline 3 & $6 \mathrm{~g}$ & $2 \mathrm{~d} 2 \mathrm{~b} 4 \mathrm{~g} 2 \mathrm{c} 2 \mathrm{a}$ \\
\hline 4 & $4 \mathrm{f}$ & $4 \mathrm{~g} 4 \mathrm{~g}$ \\
\hline 5 & $4 \mathrm{e}$ & $4 \mathrm{~g} 4 \mathrm{~g}$ \\
\hline 6 & $2 \mathrm{~d}$ & $2 \mathrm{e} 2 \mathrm{f}$ \\
\hline 7 & $2 \mathrm{c}$ & $2 \mathrm{e} 2 \mathrm{f}$ \\
\hline 8 & $2 \mathrm{~b}$ & $4 \mathrm{~g}$ \\
\hline 9 & $2 \mathrm{a}$ & $2 \mathrm{e} 2 \mathrm{f}$ \\
\hline
\end{tabular}

Table 3. Coset representatives for the right coset decomposition of $P \overline{3} 1 c$ (No. 163) with respect to $P 2 / c$ (No. 13), transformation matrix $\left(c, a-b, a+b-c ; 0,-\frac{1}{2}, 0\right)$, obtained by COSETS.

\begin{tabular}{|r|c|c|c|c|}
\hline \multicolumn{1}{|c|}{1} & 2 & 3 \\
\hline \hline$\left(\begin{array}{llll}1 & 0 & 0 & 0 \\
0 & 1 & 0 & 0 \\
0 & 0 & 1 & 0\end{array}\right)$ & $\left(\begin{array}{rrrr}1 & \frac{3}{2} & -\frac{3}{2} & \frac{3}{4} \\
0 & -\frac{1}{2} & -\frac{1}{2} & \frac{3}{4} \\
0 & \frac{3}{2} & -\frac{1}{2} & \frac{3}{4}\end{array}\right)$ & $\left(\begin{array}{rrrr}1 & -\frac{3}{2} & -\frac{3}{2} & 0 \\
0 & -\frac{1}{2} & -\frac{3}{2} & \frac{1}{2} \\
0 & -\frac{3}{2} & -\frac{1}{2} & 0\end{array}\right)$ \\
\hline$\left(\begin{array}{rrrr}-1 & 0 & 0 & \frac{1}{2} \\
0 & -1 & 0 & \frac{1}{2} \\
0 & 0 & -1 & \frac{1}{2}\end{array}\right)$ & $\left(\begin{array}{rrrr}-1 & -\frac{3}{2} & \frac{3}{2} & \frac{3}{4} \\
0 & \frac{1}{2} & \frac{1}{2} & \frac{3}{4} \\
0 & -\frac{3}{2} & \frac{1}{2} & \frac{3}{4}\end{array}\right)$ & $\left(\begin{array}{rrrr}-1 & \frac{3}{2} & \frac{3}{2} & \frac{1}{2} \\
0 & \frac{1}{2} & -\frac{1}{2} & 0 \\
0 & \frac{3}{2} & \frac{1}{2} & \frac{1}{2}\end{array}\right)$ \\
\hline
\end{tabular}

\subsection{Brillouin zones and special wave-vectors for the space groups of the arithmetic crystal class $\mathrm{mmm} \mathrm{I}$}

The following example illustrates the relation between the traditional (CDML) and the reciprocal-space group descriptions of the wave-vector types of space-groups. The included figures and tables form part of the output of the access tool KVEC.

There are four space groups belonging to the arithmetic crystal class $\mathrm{mmm} \mathrm{I:Immm} \mathrm{(No.} \mathrm{71),} \mathrm{Ibam}$ (No. 72), Ibca (No. 73) and Imma (No. 74). The wave-vector types of that arithmetic crystal class are described with respect to the corresponding reciprocal space group which is isomorphic to Fmmm (No. 69). Depending on the relations between the lattice parameters $a, b$ and $c$, there are two topologically different bodies of the Brillouin zone displayed in Fig. 2 and Fig. 3 by thin black lines; the first one has 18 vertices, 28 lines and 12 faces, while the other has 24 vertices, 34 lines and 14 faces. Simillary, the representation domains of CDML are more complicated than the asymmetric units of ITA, see Fig. 2 and Fig. 3.

In the figures, the representatives of the k-vectors symmetry points or of symmetry lines, as well as the edges of the representation domain of CDML and of the chosen asymmetric unit are brought out in colors. A k-vectors symmetry point is designated by red or cyan if it belongs to the asymmetric unit or to the representation domain of CDML. Points listed by CDML are not colored if they are part of a symmetry line or symmetry plane only. The color of the line is pink for an edge of the asymmetric unit which is not a symmetry line and it is red for a symmetry line of the asymmetric unit. The color of the line is brown with the name in red for a line which is a symmetry line as well as an edge of the asymmetric unit. The edges of the representation domains of CDML (displayed in the same figure) are colored in light blue. The corresponding symmetry points and lines are colored cyan. Edges of the representation domain or common edges of the representation domain and the asymmetric unit are 


\section{Contribution of Symmetries in Condensed Matter}

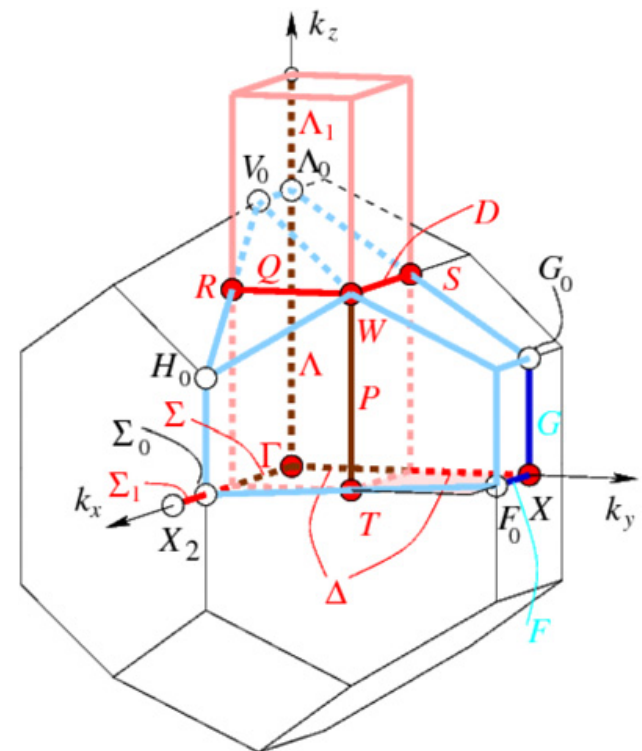

(C)bilbao crystallographic server http://www.cryst.ehu.es

Figure 2. Brillouin zone, asymmetric unit and representation domain of CDML for the arithmetic crystal class $m m m I b>a>c$ or $b>c>a$ : space groups Immm (No. 71) to Imma (No. 74); reciprocal space group Fmmm* (No. 69): $b^{*}<a^{*}<c^{*}$ or $b^{*}<c^{*}<a^{*}$. The representation domain of CDML is different from the asymmetric unit.

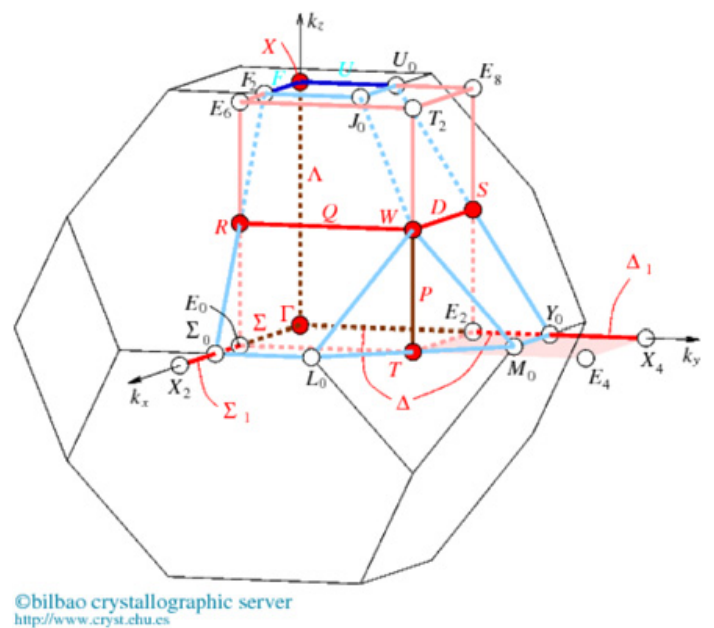

Figure 3. Brillouin zone, asymmetric unit and representation domain of CDML for the arithmetic crystal class $m m m I c>b>a$ or $c>a>b$ : space groups Immm (No. 71) to Imma (No. 74); reciprocal space group Fmmm* (No. 69): $c^{*}<b^{*}<a^{*}$ or $c^{*}<a^{*}<b^{*}$. The representation domain of CDML is different from the asymmetric unit.

colored dark blue with the letters in cyan if they are symmetry lines of the representation domain but not of the asymmetric unit.

To save space we have included only part of the list of $\mathbf{k}$-vector relations for the arithmetic crystal class $\mathrm{mmm} I$ in the table shown in Fig. 4. Each k-vector type is specified by its label and parameters. The corresponding Wyckoff positions are described by their Wyckoff letters, multiplicities, and 


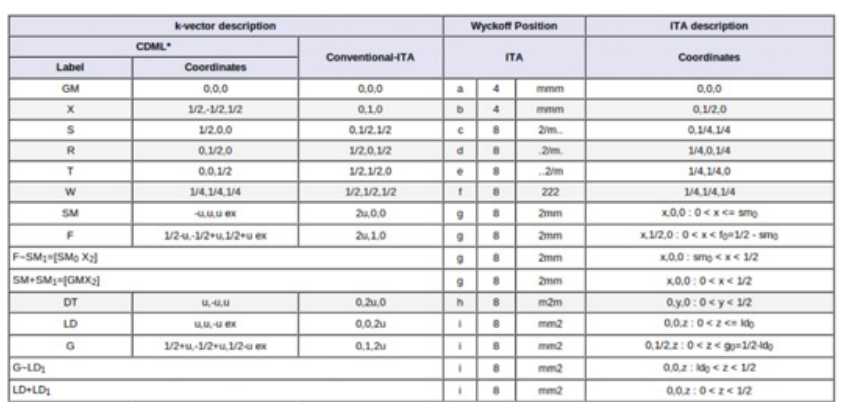

Figure 4. List of k-vector types (selection) for the arithmetic crystal class $m m I c>b>a$ or $c>a>b$ : space groups Immm (No. 71) to Imma (No. 74); reciprocal-space group $\mathrm{Fmmm}^{*}$ (No. 69) (Fig. 2).

Table 4. Structure data for $\mathrm{KAsF}_{6}, \mathrm{CsSbF}_{6}, \mathrm{BaSnF}_{6}$ and $\mathrm{KCrF}_{6}$ in rhombohedral $R \overline{3}$ (No. 148) phase.

\begin{tabular}{|ccccc|cccccc|}
\hline \multicolumn{4}{|c|}{$\mathrm{KAsF}_{6}: \mathrm{a}=7.3480, \mathrm{c}=7.2740$} & & \multicolumn{5}{c|}{$\mathrm{BaIrF}_{6}: \mathrm{a}=7.3965, \mathrm{c}=7.2826$} \\
\hline $\mathrm{K}$ & $3 \mathrm{~b}$ & $\frac{1}{3}$ & $\frac{2}{3}$ & $\frac{1}{6}$ & & $\mathrm{Ba}$ & $3 \mathrm{~b}$ & $\frac{1}{3}$ & $\frac{2}{3}$ & $\frac{1}{6}$ \\
\hline $\mathrm{As}$ & $3 \mathrm{a}$ & 0 & 0 & 0 & & $\mathrm{Ir}$ & $3 \mathrm{a}$ & 0 & 0 & 0 \\
\hline $\mathrm{F}$ & $18 \mathrm{f}$ & 0.1292 & 0.2165 & 0.1381 & & $\mathrm{~F}$ & $18 \mathrm{f}$ & 0.0729 & 0.2325 & 0.1640 \\
\hline \multicolumn{3}{|c|}{$\mathrm{BaSnF}_{6}: \mathrm{a}=7.4279, \mathrm{c}=7.4180$} & & \multicolumn{4}{c|}{$\mathrm{KCrF}_{6}: \mathrm{a}=7.3315, \mathrm{c}=7.2148$} \\
\hline $\mathrm{Ba}$ & $3 \mathrm{a}$ & 0 & 0 & 0 & & $\mathrm{~K}$ & $3 \mathrm{a}$ & 0 & 0 & 0 \\
\hline $\mathrm{Sn}$ & $3 \mathrm{~b}$ & 0 & 0 & $\frac{1}{2}$ & & $\mathrm{Cr}$ & $3 \mathrm{~b}$ & 0 & 0 & $\frac{1}{2}$ \\
\hline $\mathrm{F}$ & $18 \mathrm{f}$ & 0.2586 & 0.8262 & 0.0047 & & $\mathrm{~F}$ & $18 \mathrm{f}$ & 0.1324 & -0.0896 & 0.6420 \\
\hline
\end{tabular}

site-symmetry groups. Symmetry points, lines or planes of symmetry of CDML which correspond to the same Wyckoff position, are grouped together. The wave-vector parameters of CDML (second column) of the table in Fig. 4 are different from those of ITA (last column) because in CDML the data are always referred to a primitive basis, whereas in ITA they are referred to a centered basis. The parameter ranges (last column) are chosen such that each $\mathbf{k}$-vector orbit is represented exactly once.

It is evident from the table given in Fig. 4 that different $\mathbf{k}$ labels of CDML (first column) may belong to the same type of $\mathbf{k}$-vectors, i.e. they give rise to the same type of irreps. Due to the special shape of the representation domain of CDML, the special wave-vector line corresponding to the Wyckoff position $4 \mathrm{i}$ ( $m 2 m$ ) (fourth column) is split into two parts: $L D$ and $G$. In the ITA description $L D \cup F$ corresponds to one line $(0,0, z)$, with $0<z<\frac{1}{2}$. The splitting of the $8 \mathrm{i}$ line into two parts is a consequence of the Brillouin-zone shape for the specific values of the lattice parameters. This is confirmed from Fig. 3 where the corresponding special line $L D$ is not split.

\subsection{Comparison of Structures}

The four structures of $\mathrm{KAsF}_{6}$ [32], $\mathrm{BaIrF}_{6}$ [33], $\mathrm{BaSnF}_{6}$ [34] and $\mathrm{KCrF}_{6}$ [35] of symmetry $R \overline{3}$ (No. 148) have been reported in the literature with parameters quoted in Table 4.

According to Inorganic Structure Database (abbreviated as ICSD) [36] the four structures belong to the set of compounds with the same $\mathrm{ANX}$ formula given by $\mathrm{ABX}_{6}$. As can be seen from their graphic presentation in Fig. 5 the cell origins of the structures $\mathrm{KAsF}_{6}$ and $\mathrm{BaIrF}_{6}$ are located at octahedral sites, while the origins of $\mathrm{BaSnF}_{6}$ and $\mathrm{KCrF}_{6}$ correspond to a single atom of type $\mathrm{A}$. There is a small difference in the orientations of the octahedra, as well.

The identical Pearson symbols ${ }^{1}$ and the same sequence of occupied Wyckoff positions of the four structures show that they belong to the same isopointal structure type. ${ }^{2}$ However, the identical

\footnotetext{
1 The Pearson symbol is a three position symbol consisting of a letter indicating the crystal system, a letter for the centring type of the Bravais lattice and the total number of the atoms in the unit cell occupies the third position of the symbol.

2 The criteria for isopointal structure types are equivalent space groups, equivalent Wyckoff sequence and Pearson symbols. The subdivision of isopointal structure types into isoconfigurational structure types is done according to several criteria including
} 


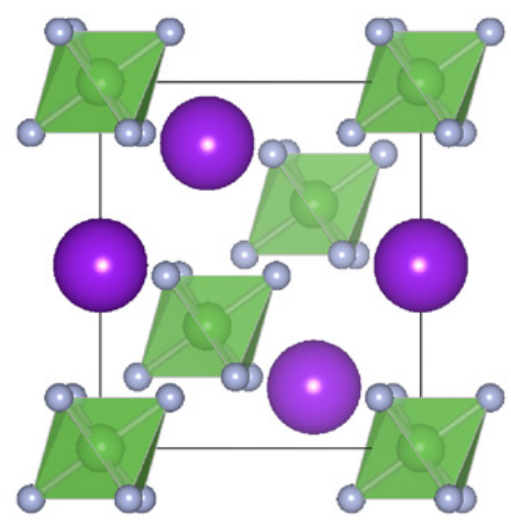

(a)

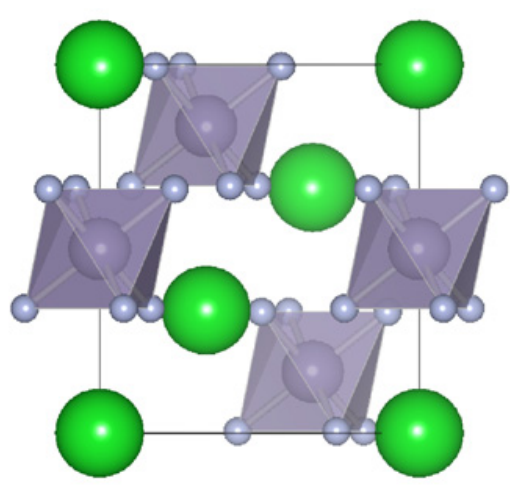

(c)

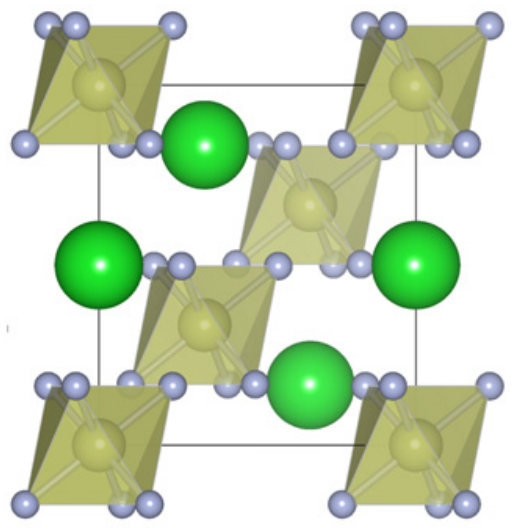

(b)

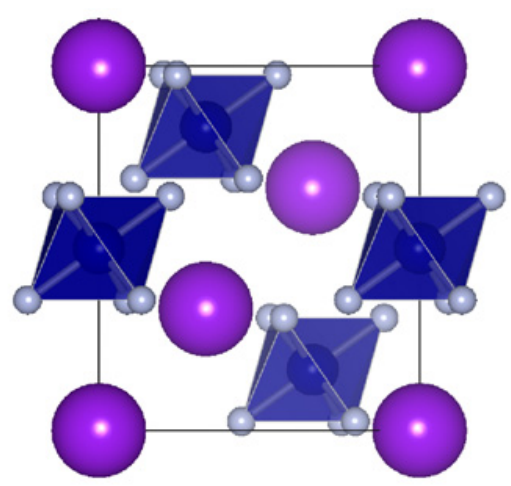

(d)

Figure 5. Visualizations of the structures (a) $\mathrm{KAsF}_{6}$, (b) $\mathrm{BaIrF}_{6}$, (c) $\mathrm{BaSnF}_{6}$ and (d) $\mathrm{KCrF}_{6}$ as defined in Table 4 projected in [100]. (Images prepared via VESTA visualization software [38]).

ANX formula and the similarity between the lattice parameters suggest also the possibility that all structures belong to the same isoconfigurational structure type and that the obvious differences in the corresponding atomic coordinates are related to different equivalent descriptions of the same structure type. We employ COMPSTRU to check the similarity of the four structures.

Since COMPSTRU assumes that the given structures have the same composition, we label the group of atoms $(\mathrm{K}, \mathrm{Ba})$ as $\mathrm{A}$, and $(\mathrm{As}, \mathrm{Ir}, \mathrm{Sn}, \mathrm{Cr})$ as $\mathrm{B}$. By selecting $\mathrm{KAsF}_{6}$ as the reference structure, we introduce successively each of the remaining three structures as the second one in COMPSTRU. The program looks through all possible equivalent descriptions of the second structure and finds the one that best fits the reference structure description, i.e. that of $\mathrm{KAsF}_{6}$. The difference between the different descriptions is quantified by the maximum distance observed between corresponding atoms in the two structures (assuming that the unit cell is unchanged, i.e. the cell strain between the two structures is not quantified).

The maximum atomic distances $\Delta$ of $\mathrm{BaIrF}_{6}, \mathrm{BaSnF}_{6}$ and $\mathrm{KCrF}_{6}$ with respect to reference structure $\mathrm{KAsF}_{6}$ together with the normalizer transformations generating the most similar equivalent descriptions

the ANX formula, $c / a$ ratio and atomic coordinates. For definition and detailed discussion of (isopointal and isoconfigurational) structure types, the reader is referred to Ref. [37]. 
Table 5. Equivalent structure data for $\mathrm{KAsF}_{6}, \mathrm{BaIrF}_{6}, \mathrm{BaSnF}_{6}$ and $\mathrm{KCrF}_{6}$ in rhombohedral $R \overline{3}$ (No. 148) phase, calculated with COMPSTRU.

\begin{tabular}{|ccccc|c|ccccc|}
\hline \multicolumn{4}{|c|}{ KAsF $_{6}$} & \multicolumn{5}{c|}{ BaIrF $_{6}: \Delta=0.2710$} \\
\hline \multicolumn{3}{|c|}{ Reference Structure } & \multicolumn{5}{c|}{ Transformation: $-\mathrm{y},-\mathrm{x}, \mathrm{z}$} \\
\hline $\mathrm{K}$ & $3 \mathrm{~b}$ & $\frac{1}{3}$ & $\frac{2}{3}$ & $\frac{1}{6}$ & & $\mathrm{Ba}$ & $3 \mathrm{~b}$ & $\frac{1}{3}$ & $\frac{2}{3}$ & $\frac{1}{6}$ \\
\hline $\mathrm{As}$ & $3 \mathrm{a}$ & 0 & 0 & 0 & & $\mathrm{Ir}$ & $3 \mathrm{a}$ & 0 & 0 & 0 \\
\hline $\mathrm{F}$ & $18 \mathrm{f}$ & 0.1292 & 0.2165 & 0.1381 & & $\mathrm{~F}$ & $18 \mathrm{f}$ & 0.1596 & 0.2325 & 0.1640 \\
\hline \multicolumn{3}{|c|}{ BaSnF $_{6}: \Delta=0.2603$} & & \multicolumn{5}{c|}{$\mathrm{KCrF}_{6}: \Delta=0.0452$} \\
\hline \multicolumn{4}{|c|}{ Transformation: $\mathrm{x}, \mathrm{y}, \mathrm{z}+\frac{1}{2}$} & & \multicolumn{5}{c|}{ Transformation: $-\mathrm{y},-\mathrm{x}, \mathrm{z}+\frac{1}{2}$} \\
\hline $\mathrm{Ba}$ & $3 \mathrm{~b}$ & $\frac{1}{3}$ & $\frac{2}{3}$ & $\frac{1}{6}$ & & $\mathrm{~K}$ & $3 \mathrm{~b}$ & $\frac{1}{3}$ & $\frac{2}{3}$ & $\frac{1}{6}$ \\
\hline $\mathrm{Sn}$ & $3 \mathrm{a}$ & 0 & 0 & 0 & & $\mathrm{Cr}$ & $3 \mathrm{a}$ & 0 & 0 & 0 \\
\hline $\mathrm{F}$ & $18 \mathrm{f}$ & 0.1595 & 0.2343 & 0.1620 & & $\mathrm{~F}$ & $18 \mathrm{f}$ & 0.1324 & 0.2220 & 0.1420 \\
\hline
\end{tabular}

Table 6. Irrep decomposition of the mechanical representations for the Wyckoff positions $2 a, 2 b, 4 c, 4 d, 4 e$ y $8 g$ of the space group $I 4 / \mathrm{mmm}$. The number of Raman $\left(A_{1 g}, B_{1 g}\right.$ y $\left.E_{g}\right)$ and infrared ( $A_{2 u}$ y $\left.E_{u}\right)$ active modes is equal to the multiplicity (indicated for each irrep in the table) multiplied by the dimension of the corresponding irrep.

\begin{tabular}{|c|cccccccccc|}
\hline $\mathrm{WP}$ & $A_{1 g}$ & $A_{1 u}$ & $A_{2 g}$ & $A_{2 u}$ & $B_{1 g}$ & $B_{1 u}$ & $B_{2 g}$ & $B_{2 u}$ & $E_{u}$ & $E_{g}$ \\
\hline \hline $8 g$ & 1 & - & - & 1 & 1 & - & - & 1 & 2 & 2 \\
$4 e$ & 1 & - & - & 1 & - & - & - & - & 1 & 1 \\
$4 d$ & - & - & - & 1 & 1 & - & - & - & 1 & 1 \\
$4 c$ & - & - & - & 1 & - & - & - & 1 & 2 & - \\
$2 b$ & - & - & - & 1 & - & - & - & - & 1 & - \\
$2 a$ & - & - & - & 1 & - & - & - & - & 1 & - \\
\hline
\end{tabular}

are given Table 5. We see that, even though the initial structures appeared to be different from each other, they were just different descriptions of the same structure type designated by $\mathrm{KOsF}_{6}$ in ICSD.

\subsection{Infrared and Raman activity in aurivillius compounds}

The Aurivillius family contains many ferroelectric compounds with the general formula of $\mathrm{Bi}_{2} \mathrm{~A}_{n-1} \mathrm{~B}_{n} \mathrm{O}_{3 n+3}$ with $\mathrm{n}=1,2,3,4$; A cation being $\mathrm{Na}^{+}, \mathrm{K}^{+}, \mathrm{Ca}^{2+}$ (coordination of 12) and $\mathrm{B}$ of the type $\mathrm{Fe}^{3+}, \mathrm{Cr}^{3+}, \mathrm{Ti}^{4+}, \mathrm{Nb}^{5+}$ (atoms with coordination of 6 ). The ideal structure at high temperatures is observed in space group $I 4 / \mathrm{mmm}$ (No. 139) and the occupied Wyckoff positions for the case $\mathrm{n}=2$ (e.g. $\mathrm{SrBi}_{4} \mathrm{Ta}_{4} \mathrm{O}_{9}$ [39]) are $2 a, 2 b, 4 d, 4 e$ and $8 g$. For the case $\mathrm{n}=3$, the $4 c$ position is also occupied.

Using the space group and occupied Wyckoff positions information, SAM first calculates the socalled mechanical representation $R$ (known also as vibrational representation) for each of the relevant Wyckoff positions. In general, the mechanical representation is a reducible one and it is decomposed into irreducible constituents. The irrep decompositions of the mechanical representations obtained by SAM for each of the Wyckoff positions $2 a, 2 b, 4 c, 4 d, 4 e$ and $8 g$ of $I 4 / \mathrm{mmm}$ are shown in Table 6. For example, the irrep decomposition of the mechanical representation for atoms occupying an orbit of the Wyckoff position $8 g$ is of the type: $R^{8 g} \sim A_{1 g} \oplus A_{2 u} \oplus B_{1 g} \oplus B_{2 u} \oplus 2 E_{g} \oplus 2 E_{u}$.

The symmetry-adapted modes are calculated applying the method of projective operators. The number of the symmetry modes for a given irrep follows from its multiplicity in the mechanical representation ( $c f$. Table 6) and the irrep dimension. For example, each of the two-dimensional irreps $E_{g}$ and $E_{u}$ appears with multiplicity two in the decomposition of $R^{8 g}$, and the corresponding four symmetry adapted modes for atoms of an $8 g$ orbit are listed in Table 7 . The computed symmetry-adapted modes are mutually orthogonal.

The infrared active modes are symmetry-adapted modes transforming according to the vector representation $V \sim A_{2 u} \oplus E_{u}$ of $I 4 / \mathrm{mmm}$. The transformation properties of the Raman-active 


\section{Contribution of Symmetries in Condensed Matter}

Table 7. Symmetry adapted modes for atoms occupying an orbit of the Wyckoff position $8 g$ of the space group $I 4 / \mathrm{mmm}$. The displacement vector components at each atomic position of the $8 \mathrm{~g}$ orbit are determined with respect to orthogonal basis.

\begin{tabular}{|c|c|c|c|c|c|c|c|c|c|c|c|c|c|}
\hline WP & Coordinates & $A_{1 g}$ & $A_{2 u}$ & $B_{1 g}$ & $B_{2 u}$ & \multicolumn{3}{|c|}{$E_{u}$} & \multicolumn{3}{|c|}{$\mathrm{E}_{g}$} \\
\hline \hline \multirow{5}{*}{$8 g$} & $(0,1 / 2, z)$ & 001 & 001 & 001 & 001 & 100 & 000 & 000 & 010 & 000 & 100 & 010 & 000 \\
& $(1 / 2,0, z)$ & 001 & 001 & $00 \overline{1}$ & $00 \overline{1}$ & 000 & 010 & 100 & 000 & 010 & 000 & 000 & 100 \\
& $(0,1 / 2, \bar{z})$ & $00 \overline{1}$ & 001 & $00 \overline{1}$ & 001 & 100 & 000 & 000 & 010 & 000 & $\overline{1} 00$ & $0 \overline{1} 0$ & 000 \\
& $(1 / 2,0, \bar{z})$ & $00 \overline{1}$ & 001 & 001 & $00 \overline{1}$ & 000 & 010 & 100 & 000 & $0 \overline{1} 0$ & 000 & 000 & $\overline{1} 00$ \\
\hline
\end{tabular}

Table 8. Structure information for $\mathrm{BaTiO}_{3}$ in space group setting $\mathrm{Amm} 2$ (No. 38).

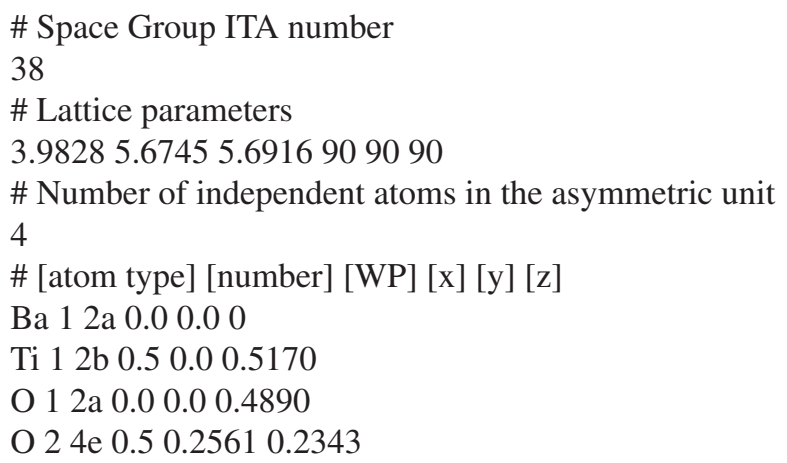

modes are determined by the irreps of the decomposition of the symmetrized square of the vector representation: $\left[V^{2}\right] \sim A_{1 g} \oplus B_{1 g} \oplus E_{g}$. For this example there is no such mode that is both infrared and Raman active, which is a common property for compounds of centrosymmetric space groups.

\subsection{Analysis of the relations between a high- and low-symmetry structure pair}

Suppose that, in an experiment, a structure with parameters given in Table 8 ( $A m m 2$, No. 38 ) is observed and we are interested in measuring its degree of structural pseudosymmetry. In other words, we want to know if the observed structure can be considered as a distorted phase of a higher symmetry structure. If the distortion is small enough, this higher symmetry structure could manifest itself as a result of a transition at higher temperature.

PSEUDO can be used to find this prototype structure and obtain a quantitative value of the required distortion. Assuming we have no information for the possible high symmetry phase, we start our search among the minimal supergroups, i.e. the first option in PSEUDO, with the default tolerance of $\Delta=2$. In the next page, PSEUDO fetches the list of the minimal supergroups of $A m m 2$, including the transformation matrices $(\boldsymbol{P}, \boldsymbol{p})$ relating the group-supergroup pairs. For each of these minimal supergroups, it also calculates the transformed cell in the supergroup basis and checks the Wyckoff splitting compatibility in regard with the transformation $(\boldsymbol{P}, \boldsymbol{p})$. The minimal supergroups fulfilling the Wyckoff splitting compatibility are pre-selected in the proposed list (in our example, Cmmm (No. 65) and $P \overline{6} 2 m$ (No. 189)). Proceeding with the calculations, PSEUDO finds a high symmetry $C \mathrm{mmm}$ structure which is within the tolerance range (having $\Delta_{\max }=0.1935$ ), whereas no structure of $P \overline{6} 2 m$ symmetry could be detected within the allowed tolerance. Furthermore, the program also gives the idealized high symmetry structure both in the subgroup and the supergroup setting. As reproduced in Table 9, distortions of each representative atom are listed in detail in terms of their displacements.

The iterative executions of PSEUDO, follow the chain of minimal supergroups

$\mathrm{Ammm} 2 \longrightarrow \mathrm{Cmmm} \longrightarrow \mathrm{P} 4 / \mathrm{mmm} \longrightarrow \mathrm{Pm} \overline{3} \mathrm{~m}$

where the associated transformation matrices $(\boldsymbol{P}, \boldsymbol{p})_{i}$ and the resulting $\left(\Delta_{\max }\right)_{i}$ values are summarized in Table 10 . 
EPJ Web of Conferences

Table 9. Displacements with regard to the idealized high symmetry structure $(\mathrm{Cmmm}$ (No. 65)) and the orthorhombic structure $\left(A m m 2\right.$ (No. 38)) of $\mathrm{BaTiO}_{3}\left(\mathbf{u}_{x, y, z}\right.$ given in relative units; lul in $\AA$ ).

\begin{tabular}{|c|c|c|c|c|c|}
\hline Atom & Idealized Coordinates & $\mathbf{u}_{x}$ & $\mathbf{u}_{y}$ & $\mathbf{u}_{z}$ & \multicolumn{1}{c|}{$|\mathbf{u}|$} \\
\hline \hline $\mathrm{Ba}$ & $(0.0000,0.0000,0.0000)$ & 0.000000 & 0.000000 & 0.000000 & 0.000000 \\
\hline $\mathrm{Ti}$ & $(0.5000,0.0000,0.5000)$ & 0.000000 & 0.000000 & 0.017000 & 0.0968 \\
\hline $\mathrm{O} 1$ & $(0.0000,0.0000,0.5000)$ & 0.000000 & 0.000000 & -0.011000 & 0.0626 \\
\hline $\mathrm{O} 2$ & $(0.5000,0.2500,0.2500)$ & 0.000000 & 0.006100 & -0.015700 & 0.0958 \\
\hline
\end{tabular}

Table 10. Transformation matrices $(\boldsymbol{P}, \boldsymbol{p})_{i}$ and $\left(\Delta_{\max }\right)_{i}$ values for the iterative executions of PSEUDO in the pseudosymmetry search of $A m m 2$ structure of $\mathrm{BaTiO}_{3}$.

\begin{tabular}{|l|c|c|}
\hline Supergroup & Transformation & $\Delta_{\max }$ \\
\hline \hline$P m \overline{3} m$ (No. 221) & a,b,c;0,0,0 & 0 \\
\hline$P 4 / m m m$ (No. 123) & a-b,a+b,c;0,0,0 & 0 \\
\hline$C m m m$ (No. 65) & c,a,b;0,0,0 & 0.1935 \\
\hline$A m m m 2$ (No. 38) & - & - \\
\hline
\end{tabular}

Table 11. The high-symmetry structure of symmetry $P m \overline{3} m$ (No. 221) corresponding to the PSEUDO result obtained in Example 3.5.

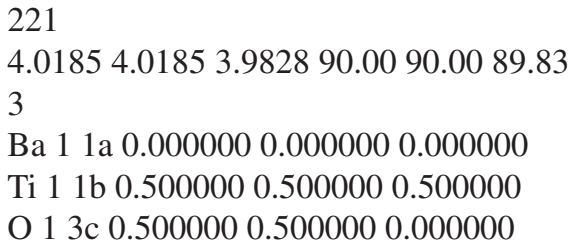

So, we have found out that the observed structure is pseudosymmetric and that the space group of its prototype high symmetry structure is $P m \overline{3} m$ (No. 221) as given in Table 11, with the transformation matrix $(\boldsymbol{P}, \boldsymbol{p})$ for the high- and low-symmetry structures being the multiplication of the transformation matrices $(\boldsymbol{P}, \boldsymbol{p})_{i}$ relating the intermediate structures: if $(\boldsymbol{P}, \boldsymbol{p})_{1}=(c, a, b ; 0,0,0)$ describes the step $A m m m 2 \longrightarrow C m m m,(\boldsymbol{P}, \boldsymbol{p})_{2}=(a-b, a+b, c ; 0,0,0)-$ the step $C m m m \longrightarrow P 4 / \mathrm{mmm}$ and $(\boldsymbol{P}, \boldsymbol{p})_{3}=(a, b, c ; 0,0,0)$ - the step $P 4 / m m m \longrightarrow P m \overline{3} m$, then the transformation matrix for $A m m m 2 \longrightarrow P m \overline{3} m$ is given by $(\boldsymbol{P}, \boldsymbol{p})=(\boldsymbol{P}, \boldsymbol{p})_{3}(\boldsymbol{P}, \boldsymbol{p})_{2}(\boldsymbol{P}, \boldsymbol{p})_{1}=(c, a-b, a+b ; 0,0,0)$.

Note that, in the output reproduced in Table 11 the lattice parameters' compatibility with the supergroup symmetry is not taken into account, being exactly the result of the transformation of the unit cell. In general, if the initial and the pseudosymmetry space group belong to different crystal families, then an accompanying symmetry breaking strain will be present.

If there is any hint about the possible pseudosymmetry of the studied structure with respect to a specific supergroup of the structure's space group, one could use the 3rd option of the PSEUDO interface where the supergroup and the transformation matrix are needed as input parameters. In this case, PSEUDO searches for pseudosymmetry directly with respect to the designated supergroup, and if successful, yields the symmetrized structure.

We can further analyze the distortions relating these two structures in terms of symmetry modes analysis applying the SYMMODES and AMPLIMODES programs. For detailed explanations of both programs with illustrative examples of their applications, the reader is referred to the article on symmetry considerations in structural phase transitions in this volume [14].

\section{SOME DEFINITIONS AND INFORMATION ON NOTATION}

\subsection{Crystallographic symmetry operations}

The notation of the space-group symmetry operations applied in the Bilbao Crystallographic Server follows closely the conventions adopted in ITA. 


\section{Contribution of Symmetries in Condensed Matter}

In order to describe the symmetry operations analytically one introduces a coordinate system $\{O, \mathbf{a}, \mathbf{b}, \mathbf{c}\}$, consisting of a set of basis vectors $\mathbf{a}, \mathbf{b}, \mathbf{c}$ and an origin $O$. A symmetry operation can be regarded as an instruction of how to calculate the coordinates $\tilde{x}, \tilde{y}, \tilde{z}$ of the image point $\tilde{X}$ from the coordinates $x, y, z$ of the original point $X$.

The equations are

$$
\begin{aligned}
& \tilde{x}=W_{11} x+W_{12} y+W_{13} z+w_{1} \\
& \tilde{y}=W_{21} x+W_{22} y+W_{23} z+w_{2} \\
& \tilde{z}=W_{31} x+W_{32} y+W_{33} z+w_{3},
\end{aligned}
$$

These equations can be written using the matrix formalism:

$$
\tilde{\boldsymbol{x}}=\boldsymbol{W} \boldsymbol{x}+\boldsymbol{w}=(\boldsymbol{W}, \boldsymbol{w}) \boldsymbol{x} \quad \text { where }
$$

the symmetry operations $(\boldsymbol{W}, \boldsymbol{w})$ are given in a matrix-column form consisting of a $(3 \times 3)$ matrix (linear) part $\boldsymbol{W}$ and a $(3 \times 1)$-column(translation) part $\boldsymbol{w}$ :

$$
(\boldsymbol{W}, \boldsymbol{w})=\left(\begin{array}{lll|l}
W_{11} & W_{12} & W_{13} & w_{1} \\
W_{21} & W_{22} & W_{23} & w_{2} \\
W_{31} & W_{32} & W_{33} & w_{3}
\end{array}\right)
$$

Apart from the matrix-column pair presentation of $(\boldsymbol{W}, \boldsymbol{w})$ often the so-called short-hand notation for the symmetry operations is used. It is obtained from the left-hand side of equ. (4.1) by omitting the terms with coefficients 0 and writing in one line the three different rows of equ. (4.1), separated by commas. For example, consider the symmetry operation under No. 30 in the list of general positions obtained by GENPOS for the space group $\operatorname{Pn} \overline{3} n$, No. 222 (origin choice 2):

$$
\tilde{\boldsymbol{x}}=(\boldsymbol{W}, \boldsymbol{w}) \boldsymbol{x}=\left(\begin{array}{ccc}
0 & 0 & -1 \\
1 & 0 & 0 \\
0 & 1 & 0
\end{array}\right)\left(\begin{array}{l}
x \\
y \\
z
\end{array}\right)+\left(\begin{array}{c}
0 \\
1 / 2 \\
1 / 2
\end{array}\right) \text { would be }
$$

$\tilde{x}=0 x+0 y-1 z, \tilde{y}=1 x+0 y+0 z+1 / 2, \tilde{z}=0 x+1 y+0 z+1 / 2$.

The shorthand notation of $(\boldsymbol{W}, \boldsymbol{w})$ reads: $\bar{z}, x+1 / 2, y+1 / 2$.

\subsection{Coordinate transformations: basic results}

Let a coordinate system be given with a basis $\left(\mathbf{a}_{1}, \mathbf{a}_{2}, \mathbf{a}_{3}\right)$ and an origin $O$. The general transformation (affine transformation) of the coordinate system consists of two parts, a linear part and a shift of the origin. The transformation is uniquely defined by the $(3 \times 3)$ matrix $\boldsymbol{P}$ of the linear part and the $(3 \times 1)$ column matrix $\boldsymbol{p}$ containing the components of the shift vector $\boldsymbol{p}$.

1. The linear part is described by a $(3 \times 3)$ matrix

$$
\boldsymbol{P}=\left(\begin{array}{lll}
P_{11} & P_{12} & P_{13} \\
P_{21} & P_{22} & P_{23} \\
P_{31} & P_{32} & P_{33}
\end{array}\right)
$$

i.e. the matrix which relates the new basis $\left(\mathbf{a}_{1}^{\prime}, \mathbf{a}_{2}^{\prime}, \mathbf{a}_{3}^{\prime}\right)$ to the old basis $\left(\mathbf{a}_{1}, \mathbf{a}_{2}, \mathbf{a}_{3}\right)$ according to

$$
\left(\mathbf{a}_{1}^{\prime}, \mathbf{a}_{2}^{\prime}, \mathbf{a}_{3}^{\prime}\right)=\left(\mathbf{a}_{1}, \mathbf{a}_{2}, \mathbf{a}_{3}\right) \boldsymbol{P}=\left(\mathbf{a}_{1}, \mathbf{a}_{2}, \mathbf{a}_{3}\right)\left(\begin{array}{lll}
P_{11} & P_{12} & P_{13} \\
P_{21} & P_{22} & P_{23} \\
P_{31} & P_{32} & P_{33}
\end{array}\right) .
$$

2. A shift of the origin is defined by the shift vector

$$
\boldsymbol{p}=\left(p_{1} \mathbf{a}_{1}, p_{2} \mathbf{a}_{2}, p_{3} \mathbf{a}_{3}\right)
$$




\section{EPJ Web of Conferences}

The basis vectors $\mathbf{a}_{1}, \mathbf{a}_{2}, \mathbf{a}_{3}$ are fixed at the origin $O$; the new basis vectors $\left(\mathbf{a}_{1}^{\prime}, \mathbf{a}_{2}^{\prime}, \mathbf{a}_{3}^{\prime}\right)$ are fixed at the new origin $O^{\prime}$ that has the coordinates $\left(p_{1}, p_{2}, p_{3}\right)$ in the old coordinate system.

The general affine transformation of the coordinates of a point $X$ in direct space (given by the column $\left.\boldsymbol{x}=\left(x_{1}, x_{2}, x_{3}\right)\right)$ is given by the following formula:

$$
\boldsymbol{x}^{\prime}=(\boldsymbol{P}, \boldsymbol{p})^{-1} \boldsymbol{x}=\boldsymbol{P}^{-1} \boldsymbol{x}-\boldsymbol{P}^{-1} \boldsymbol{p}=\boldsymbol{P}^{-1}(\boldsymbol{x}-\boldsymbol{p}) .
$$

The metric tensor $\boldsymbol{G}$ of the unit cell in direct lattice is transformed by the matrix $\boldsymbol{P}$ as follows:

$$
\boldsymbol{G}^{\prime}=\boldsymbol{P}^{t} \boldsymbol{G P}
$$

where $\boldsymbol{P}^{t}$ is the transposed matrix of $\boldsymbol{P}$.

The volume of the unit cell $V$ changes with the transformation. The volume of the new unit cell $V^{\prime}$ is obtained by

$$
V^{\prime}=\operatorname{det}(\boldsymbol{P}) V
$$

with $\operatorname{det}(\boldsymbol{P})$ being the determinant of the matrix $\boldsymbol{P}$.

Also, the matrix-column pairs of the symmetry operations are changed by a change of the coordinate system. If a symmetry operation is described in the "old"(unprimed) coordinate system by the matrixcolumn pair $(\boldsymbol{W}, \boldsymbol{w})$ and in the "new" (primed) coordinate system by the pair $\left(\boldsymbol{W}^{\prime}, \boldsymbol{w}^{\prime}\right)$, then the relation between the pairs $(\boldsymbol{W}, \boldsymbol{w})$ and $\left(\boldsymbol{W}^{\prime}, \boldsymbol{w}^{\prime}\right)$ is given by:

$$
\left(\boldsymbol{W}^{\prime}, \boldsymbol{w}^{\prime}\right)=(\boldsymbol{P}, \boldsymbol{p})^{-1}(\boldsymbol{W}, \boldsymbol{w})(\boldsymbol{P}, \boldsymbol{p})
$$

The coordinate systems of the space groups used by the programs and database on the Bilbao Crystallographic Server (referred to as standard or default settings) for the presentation of the spacegroup data coincide with the conventional space-group descriptions found in ITA. For space groups with more than one description in ITA, the following settings are chosen as standard: unique axis $b$ setting, cell choice 1 for monoclinic groups, hexagonal axes setting for rhombohedral groups, and origin choice 2 (origin in $\overline{1}$ ) for the centrosymmetric groups listed with respect to two origins in ITA. Optionally certain applications allow the usage of the so-called ITA settings which include all conventional settings applied in ITA (e.g. rhombohedral axes setting for rhombohedral groups, and origin choice 1 for the centrosymmetric groups) and the great variety of about 530 settings of monoclinic and orthorhombic groups listed in Table 4.3.2.1 of ITA. Settings different from the standard ones and the ITA settings are designated as non-conventional.

\subsection{Group-subgroup relations of space groups}

\subsubsection{Basic definitions}

A subset $\mathcal{H}$ of elements of a group $\mathcal{G}$ is called a subgroup of $\mathcal{G}, \mathcal{G}>\mathcal{H}$ if it fulfills the group postulates with respect to the law of composition of $\mathcal{G}$. In general, the group $\mathcal{G}$ itself is included among the set of subgroups of $\mathcal{G}$, i.e. $\mathcal{G} \geq \mathcal{H}$. If $\mathcal{G}>\mathcal{H}$ is fulfilled, then the subgroup $\mathcal{H}$ is called a proper subgroup of $\mathcal{G}$.

In a relation $\mathcal{G} \geq \mathcal{H}$ or $\mathcal{G}>\mathcal{H}, \mathcal{G}$ is called a supergroup of $\mathcal{H}$. A subgroup $\mathcal{H}<\mathcal{G}$ is a maximal subgroup if no group $\mathcal{Z}$ exists for which $\mathcal{H}<\mathcal{Z}<\mathcal{G}$ holds. If $\mathcal{H}$ is a maximal subgroup of $\mathcal{G}$, then $\mathcal{G}$ is a minimal supergroup of $\mathcal{H}$.

Let $\mathcal{H}<\mathcal{G}$ be a subgroup of $\mathcal{G}$ of order $|\mathcal{H}|$. Because $\mathcal{H}$ is a proper subgroup of $\mathcal{G}$ there must be elements $g_{q} \in \mathcal{G}$ which are not elements of $\mathcal{H}$. Let $g_{2} \in \mathcal{G}$ be one of them. Then the set of elements $g_{2} \mathcal{H}=\left\{g_{2} h_{j} \mid h_{j} \in \mathcal{H}\right\}^{3}$ is a subset of elements of $\mathcal{G}$ with the property that all its elements are different and that the sets $\mathcal{H}$ and $g_{2} \mathcal{H}$ have no element in common. Thus, also the set $g_{2} \mathcal{H}$ contains $|\mathcal{H}|$ elements of $\mathcal{G}$. If there is another element $g_{3} \in \mathcal{G}$ which does belong neither to $\mathcal{H}$ nor to $g_{2} \mathcal{H}$, one can form

\footnotetext{
3 The formulation $g_{2} \mathcal{H}=\left\{g_{2} h_{j} \mid h_{j} \in \mathcal{H}\right\}$ means: " $g_{2} \mathcal{H}$ is the set of the products $g_{2} h_{j}$ of $g_{2}$ with all elements $h_{j} \in \mathcal{H}$."
} 


\section{Contribution of Symmetries in Condensed Matter}

another set $g_{3} \mathcal{H}=\left\{g_{3} h_{j} \mid h_{j} \in \mathcal{H}\right\}$. All elements of $g_{3} \mathcal{H}$ are different and no one occurs already in $\mathcal{H}$ or in $g_{2} \mathcal{H}$. This procedure can be continued until each element $g_{r} \in \mathcal{G}$ belongs to one of these sets. In this way the group $\mathcal{G}$ can be partitioned, such that each element $g \in \mathcal{G}$ belongs to exactly one of these sets.

The partition just described is called a decomposition $(\mathcal{G}: \mathcal{H})$ into left cosets of the group $\mathcal{G}$ relative to the group $\mathcal{H}$.

$$
\mathcal{G}=\mathcal{H} \cup g_{2} \mathcal{H} \cup \ldots \cup g_{i} \mathcal{H}
$$

The sets $g_{p} \mathcal{H}, p=1, \ldots, i$ are called left cosets, because the elements $h_{j} \in \mathcal{H}$ are multiplied with the new elements from the left-hand side. The procedure is called a decomposition into right cosets $\mathcal{H} g_{s}$ if the elements $h_{j} \in \mathcal{H}$ are multiplied with the new elements $g_{s}$ from the right-hand side.

$$
\mathcal{G}=\mathcal{H} \cup \mathcal{H} g_{2} \cup \ldots \cup \mathcal{H} g_{i}
$$

The elements $g_{p}$ or $g_{s}$ are called the coset representatives. The number of cosets is called the index $[i]=|\mathcal{G}: \mathcal{H}|$ of $\mathcal{H}$ in $\mathcal{G}$.

Two subgroups $\mathcal{H}_{j}, \mathcal{H}_{k}<\mathcal{G}$ are called conjugate if there is an element $g_{q} \in \mathcal{G}$ such that $g_{q}^{-1} \mathcal{H}_{j} g_{q}=$ $\mathcal{H}_{k}$ holds. In this way, the subgroups of $\mathcal{G}$ are distributed into classes of conjugate subgroups that are also called conjugacy classes of subgroups. Subgroups in the same conjugacy class are isomorphic and thus have the same order. Different conjugacy classes of subgroups may contain different numbers of subgroups, i.e. have different lengths.

A subgroup $\mathcal{H}$ of a group $\mathcal{G}$ is a normal subgroup $\mathcal{H} \triangleleft \mathcal{G}$ if it is identical with all of its conjugates, $g_{q}^{-1} \mathcal{H} g_{q}=\mathcal{H}$, for all $g_{q} \in \mathcal{G}$, i.e. if its conjugacy class consists of the one subgroup $\mathcal{H}$ only.

\subsubsection{Subgroups of space groups}

The set of all symmetry operations of a three-dimensional crystal pattern forms its symmetry group, which is the space group of this crystal pattern. An essential feature of a crystal pattern is its periodicity which indicates that there are translations among its symmetry operations. The infinite number of translations determines the infinite order of any space group. The set of all translations of a space group $\mathcal{G}$ forms the infinite translation subgroup $\mathcal{T}(\mathcal{G}) \triangleleft \mathcal{G}$ which is a normal subgroup of $\mathcal{G}$ of finite index. Consider the right coset decomposition of $\mathcal{G}$ relative to $\mathcal{T}(\mathcal{G})$.

$$
\begin{array}{cccccc}
(\boldsymbol{I}, \boldsymbol{o}) & \left(\boldsymbol{W}_{2}, \boldsymbol{w}_{2}\right) & \ldots & \left(\boldsymbol{W}_{m}, \boldsymbol{w}_{m}\right) & \ldots & \left(\boldsymbol{W}_{i}, \boldsymbol{w}_{i}\right) \\
\left(\boldsymbol{I}, \boldsymbol{t}_{1}\right) & \left(\boldsymbol{W}_{2}, \boldsymbol{w}_{2}+\boldsymbol{t}_{1}\right) & \ldots & \left(\boldsymbol{W}_{m}, \boldsymbol{w}_{m}+\boldsymbol{t}_{1}\right) & \ldots & \left(\boldsymbol{W}_{i}, \boldsymbol{w}_{i}+\boldsymbol{t}_{1}\right) \\
\left(\boldsymbol{I}, \boldsymbol{t}_{2}\right) & \left(\boldsymbol{W}_{2}, \boldsymbol{w}_{2}+\boldsymbol{t}_{2}\right) & \ldots & \left(\boldsymbol{W}_{m}, \boldsymbol{w}_{m}+\boldsymbol{t}_{2}\right) & \ldots & \left(\boldsymbol{W}_{i}, \boldsymbol{w}_{i}+\boldsymbol{t}_{2}\right) \\
\ldots & \ldots & \ldots & \ldots & \ldots & \ldots \\
\left(\boldsymbol{I}, \boldsymbol{t}_{j}\right) & \left(\boldsymbol{W}_{2}, \boldsymbol{w}_{2}+\boldsymbol{t}_{j}\right) & \ldots & \left(\boldsymbol{W}_{m}, \boldsymbol{w}_{m}+\boldsymbol{t}_{j}\right) & \ldots & \left(\boldsymbol{W}_{i}, \boldsymbol{w}_{i}+\boldsymbol{t}_{j}\right) \\
\ldots & \ldots & \ldots & \ldots & \ldots & \ldots
\end{array}
$$

Obviously, the coset representatives of the decomposition $(\mathcal{G}: \mathcal{T}(\mathcal{G}))$ represent in a clear and compact way the infinite number of elements of the space group $\mathcal{G}$. And this is one of the ways of presenting the space groups in ITA and also in the Bilbao Crystallographic Server, i.e. by the matrices of the coset representatives of $(\mathcal{G}: \mathcal{T}(\mathcal{G}))$ listed in the General position.

Each coset in the decomposition $(\mathcal{G}: \mathcal{T}(\mathcal{G}))$ is characterized by its linear part. One can show that the set of linear parts, represented by the set of matrices $\boldsymbol{W}_{j}$, forms a group which is called the point group $\mathcal{P}_{\mathcal{G}}$ of the space group $\mathcal{G}$. The point groups which can belong to space groups are called crystallographic point groups. 


\section{EPJ Web of Conferences}

The following types of subgroups of space groups are to be distinguished [11]:

A subgroup $\mathcal{H}$ of a space group $\mathcal{G}$ is called a translationengleiche subgroup or a $t$-subgroup of $\mathcal{G}$ if the set $\mathcal{T}(\mathcal{G})$ of translations is retained, i.e. $\mathcal{T}(\mathcal{H})=\mathcal{T}(\mathcal{G})$, but the number of cosets of the decomposition $(\mathcal{G}: \mathcal{T}(\mathcal{G}))$, i.e. the order of the point group $\mathcal{P}_{\mathcal{G}}$ is reduced.

A subgroup $\mathcal{H}<\mathcal{G}$ of a space group $\mathcal{G}$ is called a klassengleiche subgroup or a $k$-subgroup if the set $\mathcal{T}(\mathcal{G})$ of all translations of $\mathcal{G}$ is reduced to $\mathcal{T}(\mathcal{H})<\mathcal{T}(\mathcal{G})$ but all linear parts of $\mathcal{G}$ are retained. Then the number of cosets of the decompositions $(\mathcal{H}: \mathcal{T}(\mathcal{H}))$ and $(\mathcal{G}: \mathcal{T}(\mathcal{G}))$ is the same, i.e. the order of the point group $\mathcal{P}_{\mathcal{H}}$ is the same as that of $\mathcal{P}_{\mathcal{G}}$.

A klassengleiche or $k$-subgroup $\mathcal{H}<\mathcal{G}$ is called isomorphic or an isomorphic subgroup if it belongs to the same affine space-group type (isomorphism type) as $\mathcal{G}$ does.

A subgroup of a space group is called general or a general subgroup if it is neither a translationengleiche nor a klassengleiche subgroup. It has lost translations as well as linear parts, i.e. point-group symmetry.

Any subgroup $\mathcal{H}$ of a group $\mathcal{G}$ is related to a specific subset of elements of $\mathcal{G}$ and this subset defines the subgroup uniquely: different subgroups of $\mathcal{G}$, even those isomorphic to $\mathcal{H}$, correspond to different subsets of the elements of $\mathcal{G}$. For example, the listing of the maximal $t$-subgroups of the space groups in ITA is based on this fact: apart from the space-group type and index, each $t$-subgroup $\mathcal{H}$ is specified by the set of coordinate triplets of the general position of $\mathcal{G}$ which are retained in $\mathcal{H}$.

In the Bilbao Crystallographic Server any subgroup $\mathcal{H}$ of a space group $\mathcal{G}$ is specified by its ITAnumber, the index in the group $\mathcal{G}$ and the transformation matrix-column pair $(\boldsymbol{P}, \boldsymbol{p})$ that relates the standard bases $(\mathbf{a}, \mathbf{b}, \mathbf{c})_{\mathcal{H}}$ of $\mathcal{H}$ and $(\mathbf{a}, \mathbf{b}, \mathbf{c})_{\mathcal{G}}$ of $\mathcal{G}$ :

$$
(\mathbf{a}, \mathbf{b}, \mathbf{c})_{\mathcal{H}}=(\mathbf{a}, \mathbf{b}, \mathbf{c})_{\mathcal{G}} \boldsymbol{P}
$$

The column $\boldsymbol{p}=\left(p_{1}, p_{2}, p_{3}\right)$ of coordinates of the origin $O_{\mathcal{H}}$ of $\mathcal{H}$ is referred to the coordinate system of $\mathcal{G}$.

The subgroup data listed in the server, i.e. the space-group type of $\mathcal{H}$ and the transformation matrix $(\boldsymbol{P}, \boldsymbol{p})$, are completely sufficient to define the subgroup uniquely: the transformation of the coordinate triplets of general-position of $\mathcal{H}$ (in standard setting) to the coordinate system of $\mathcal{G}$ by $(\boldsymbol{P}, \boldsymbol{p})^{-1}$ yields exactly the subset of elements of $\mathcal{G}$ corresponding to $\mathcal{H}$.

A very important result on group-subgroup relations between space groups is given by Hermann's theorem: For any group-subgroup chain $\mathcal{G}>\mathcal{H}$ between space groups there exists a uniquely defined space group $\mathcal{M}$ with $\mathcal{G} \geq \mathcal{M} \geq \mathcal{H}$, where $\mathcal{M}$ is a translationengleiche subgroup of $\mathcal{G}$ and $\mathcal{H}$ is a klassengleiche subgroup of $\mathcal{M}$. The decisive point is that any group-subgroup chain between space groups $\mathcal{G}>\mathcal{H}$ of index [i] can be split into a translationengleiche subgroup chain between the space groups $\mathcal{G}$ and $\mathcal{M}$ of index $\left[i_{P}\right]$ and a klassengleiche subgroup chain between the space groups $\mathcal{M}$ and $\mathcal{H}$ of index $\left[i_{L}\right]$ where $[i]=\left[i_{P}\right] \cdot\left[i_{L}\right]$. The first one, also called $t$-chain $\mathcal{G} \stackrel{i_{P}}{>} \mathcal{M}$, is related to the reduction of the point-group symmetry in the subgroup. The second one $\mathcal{M} \stackrel{i_{L}}{>} \mathcal{H}_{j}$ is known also as $k$-chain and it takes account of the loss of translations.

$\mathcal{G}>\mathcal{M}$ with $[i]=\left[i_{P}\right]$ and $\mathcal{M}>\mathcal{H}_{j}$ with $[i]=\left[i_{L}\right]$ where $[i]=\left[i_{P}\right] \cdot\left[i_{L}\right]$. The first one, the socalled translationengleiche or $t$-chain $\mathcal{G} \stackrel{i_{P}}{>} \mathcal{M}$, is related to the reduction of the point-group symmetry in the subgroup. The second one is known as klassengleiche, or $k$-chain $\mathcal{M} \stackrel{i_{L}}{>} \mathcal{H}_{j}$, and it takes account of the loss of translations.

It may happen, that either $\mathcal{G}=\mathcal{M}$ or $\mathcal{H}=\mathcal{M}$ holds. In particular, one of these equations must hold if $\mathcal{H}<\mathcal{G}$ is a maximal subgroup of $\mathcal{G}$. In other words, a maximal subgroup of a space group is either a translationengleiche subgroup or a klassengleiche subgroup, never a general subgroup.

If the maximal subgroups are known for each space group, then in principle each non-maximal subgroup of a space group $\mathcal{G}$ with finite index can be obtained from the data on maximal subgroups. 


\section{Contribution of Symmetries in Condensed Matter}

A non-maximal subgroup $\mathcal{H}<\mathcal{G}$ of finite index [i] is connected with the original group $\mathcal{G}$ through a chain $\mathcal{H}=\mathcal{Z}_{k}<\mathcal{Z}_{k-1}<\ldots<\mathcal{Z}_{1}<\mathcal{Z}_{0}=\mathcal{G}$, where each group $\mathcal{Z}_{j}<\mathcal{Z}_{j-1}$ is a maximal subgroup of $\mathcal{Z}_{j-1}$, with the index $\left[i_{j}\right]=\left|\mathcal{Z}_{j-1}: \mathcal{Z}_{j}\right|, j=1, \ldots, k$. The number $k$ is finite and the relation $i=\prod_{j=1}^{k} i_{j} \quad$ holds, i.e. the total index [i] is the product of the indices $i_{j}$.

In a similar way, one can express the transformation matrix $(\boldsymbol{P}, \boldsymbol{p})$ for the symmetry reduction $\mathcal{G} \longrightarrow \mathcal{H}$ as a product of the transformation matrices $(\boldsymbol{P}, \boldsymbol{p})_{j}$ characterizing each of the intermediate steps $\mathcal{Z}_{j-1}>\mathcal{Z}_{j}:(\boldsymbol{P}, \boldsymbol{p})=(\boldsymbol{P}, \boldsymbol{p})_{1}(\boldsymbol{P}, \boldsymbol{p})_{2} \cdots(\boldsymbol{P}, \boldsymbol{p})_{k}$ (here the matrices $(\boldsymbol{P}, \boldsymbol{p})_{j}$ relate the bases of $\mathcal{Z}_{j-1}$ and $\mathcal{Z}_{j}$, i.e. $\left.(\mathbf{a}, \mathbf{b}, \mathbf{c})_{j}=(\mathbf{a}, \mathbf{b}, \mathbf{c})_{j-1} \boldsymbol{P}_{j}\right)$.

This work has been supported by the Spanish Ministry of Science and Technology (project MAT2008-05839) and the Basque Government (project IT-282-07).

\section{References}

[1] M. I. Aroyo, A. Kirov, C. Capillas, J.M. Perez-Mato, and H. Wondratschek. Acta Crystallogr. A, 62(2):115-128, 2006.

[2] M. I. Aroyo, J. M. Perez-Mato, C. Capillas, E. Kroumova, S. Ivantchev, G. Madariaga, A. Kirov, and H. Wondratschek. Z. Kristallogr., 221(1):15-27, 2006.

[3] International Tables of Crystallography, Vol. A: Space-Group Symmetry. Edited by T. Hahn, 5th ed. (2002) Dordrecht: Kluwer Academic Publishers. [Abbreviated: ITA]

[4] International Tables for Crystallography, Vol. A1: Symmetry Relations between Space Groups. Edited by H. Wondrtaschek and U. Müller. 2nd ed. Chichester: John Wiley \& Sons, 2010. [Abbreviated: ITA1]

[5] International Tables for Crystallography, Vol. E: Subperiodic Groups. Edited by V. Kopsky, D. Litvin, 2nd ed. Chichester: John Wiley \& Sons, 2010. [Abbreviated: ITE]

[6] G. Wintgen. Math. Ann., 118: 195-215, 1941 (in German).

[7] M. I. Aroyo and H. Wondratschek. Z. Kristallogr., 210:243-254, 1995.

[8] M. I. Aroyo and H. Wondratschek. Crystallographic viewpoints in the classification of spacegroup representations. In International Tables for Crystallography, Vol. B: Reciprocal Space Edited by U. Shmueli, 3rd ed. pp. 175-192. Dordrecht: Springer, 2008.

[9] A. P. Cracknell, B. L. Davies, S. C. Miller, and W. F. Love. Kronecker Product Tables, Volume 1. General Introduction and Tables of Irreducible Representations of Space Groups. New York: IFI/Plenum, 1979. [Abbreviated: CDML]

[10] S. Ivantchev, E. Kroumova, G. Madariaga, J. M. Perez-Mato, and M. I. Aroyo. J. Appl. Crystallogr., 33(4):1190-1191, 2000.

[11] Hermann, C. Zur systematischen Strukturtheorie. IV. Untergruppen. Z. Kristallogr. 69, 533-555, 1929.

[12] E. Kroumova, J. M. Perez-Mato, and M. I. Aroyo. J. Appl. Crystallogr., 31(4):646, 1998.

[13] S. Ivantchev, E. Kroumova, M. I. Aroyo, J. M. Perez-Mato, J. M. Igartua, G. Madariaga, and H. Wondratschek. J. Appl. Crystallogr., 35(4):511-512, 2002.

[14] See chapter by Perez-Mato et al.

[15] C. Capillas, J. M. Perez-Mato, and M. I. Aroyo. J. Phys-Condens. Mat., 19(27):275203, 2007.

[16] M. I. Aroyo, J. M. Perez-Mato, D. Orobengoa, E. S. Tasci, G. de la Flor, and Kirov A. Bulg. Chem. Commun., 43(2): 183-197, 2011.

[17] S. R. Hall, F. H. Allen, and I. D. Brown. Acta Crystallogr. A, 47(6):655-685, 1991.

[18] J. L. Schlenker, G. V. Gibbs, and M. B. Boisen. Acta Crystallogr. A, 34:52-54, 1978.

[19] M. Catti. Acta Crystallogr. A, 41(5):494-500, 1985. 
[20] W.W. Schmahl. Z. Kristallogr., 191:23-38, 1990.

[21] E. Koch, W. Fischer. Z. Kristallogr., 221(1):1-14, 2006.

[22] Jmol: an open-source Java viewer for chemical structures in 3D - http://www.jmol.org/.

[23] E. Kroumova, M. I. Aroyo, J. M. Perez-Mato, A. Kirov, C. Capillas, S. Ivantchev, and H. Wondratschek. Phase Transit., 76(1-2):155-170, 2003.

[24] A. K. Kirov, M. I. Aroyo, and J. M. Perez-Mato. J. Appl. Crystallogr., 36(4):1085-1089, 2003.

[25] C. Capillas, E. Kroumova, M. I. Aroyo, J. M. Perez-Mato, H. T. Stokes, and D. M. Hatch. J. Appl. Crystallogr., 36(3 Part 2):953-954, 2003.

[26] D. Orobengoa, C. Capillas, M. I. Aroyo, and J. M. Perez-Mato. J. Appl. Crystallogr., 42(5):820833, 2009.

[27] C. Capillas, E. S. Tasci, G. de la Flor, D. Orobengoa, J. M. Perez-Mato, and M. I. Aroyo. Z. Kristallogr., 226(2):186-196, 2011.

[28] J. M. Igartua, M. I. Aroyo and J. M. Perez-Mato. Phys. Rev. B54:12744-12752, 1996.

[29] R. W. Grosse-Kunstleve. Computational crystallography toolbox - http://cctbx.sourceforge.net/.

[30] C. Capillas, M. I. Aroyo and J. M. Perez-Mato. Z. Kristallogr., 220:691-699, 2005.

[31] V. Petricek, M. Dusek, L. Palatinus Jana2006. The Crystallographic Computing System Institute of Physics, Praha, Czech Republic, 2006.

[32] J. Peterkova, M. Dusek, V. Petricek, and J. Loub. Acta Crystallogr. B, 54(6):809-818, 1998.

[33] A. I. Smolentsev, A. I. Gubanov, and A. M. Danilenko. Acta Crystallogr. C, 63:199-i101, 2007.

[34] S. Becker, G. Benner, and R. Hoppe. Z. Anorg. Allg. Chem., 591(1):7-16, 1990.

[35] Z. Mazej and E. Goreshnik. Eur. J. Inorg. Chem., 2008(11):1795-1812, 2008.

[36] G. Bergerhoff and I. D. Brown in Crystallographic Databases, F.H. Allen et al. (Hrsg.) Chester: International Union for Crystallography, 1987. [Abbreviated: ICSD]

[37] R. Allmann and R. Hinek. Acta Crystallogr. A, 63:412-417, 2007.

[38] K. Momma and F. Izumi. J. Appl. Crystallogr., 41:653-658, 2008.

[39] C. H. Hervoches, J. T. Irvine, and P. Lightfoot. Phys. Rev. B, 64:100102, 2001. 